Sädhanä, Vol. 18, Part 5, September 1993, pp. 761-786. (C) Printed in India.

\title{
Bifurcation and chaos in power systems
}

\author{
CHIN-WOO TAN, MATTHEW VARGHESE, PRAVIN VARAIYA, \\ and FELIX WU
}

Department of Electrical Engineering and Computer Sciences, University of California, Berkeley, CA 94720, USA

\begin{abstract}
A detailed example of a power system model with load dynamics is studied by investigating qualitative changes or bifurcations in its behaviour as a reactive power demand at one load bus is increased. In addition to the saddle-node bifurcation often associated with voltage collapse, we find other bifurcation phenomena which include Hopf bifurcation, cyclic fold bifurcation, period doubling bifurcation, and the emergence of chaos. The presence of these dynamic bifurcations motivates a re-examination of the role of saddle-node bifurcations in the voltage collapse phenomenon. In fact, simulation results suggest that voltage collapse may take place before the reactive power demand is increased to the system steady-state operating limit where a saddle-node bifurcation is detected. We also consider the role that the algebraic constraints imposed by some load models may play in the global analysis of the attractors of the system. Implications for power system operations are drawn.
\end{abstract}

Keywords. Chaos in power systems; bifurcations; voltage collapse phenomenon.

\section{Introduction}

In this paper a power system model that exhibits both static and dynamic bifurcations is studied. In addition, the system is shown to exhibit the period doubling route to chaotic behaviour. The onset of chaos as well as the destruction of the chaotic attractor are recorded. We point out cases of multistable behaviour where a stable equilibrium point (operating point) and other stable orbital structures (such as stable periodic orbits) exist simultaneously as the reactive power demand at the load bus is varied. Such multistable behaviour may shrink the stability region of the stable equilibrium. It is thus crucial to estimate the stability region (also called the domain of attraction) of the stable equilibrium point at parameter values where multistable behaviour persists. A "small" stability region means the system cannot sustain a "large" disturbance and the system trajectory may become oscillatory or unbounded. In recent years, voltage collapse in electric power systerns has received significant attention in the literature. This physical phenomenon is typically characterized by a period of slowly decreasing voltage followed by an accelerating collapse of voltage. Studies of voltage collapse such as those in Dobson \& Chiang (1989) and Kwatny 
et al (1986) stress the importance of sensitivity indices of the nearly singular Jacobian matrix near a saddle-node bifurcation (SNB) where the reactive power demand is increased to the system steady-state operating limit. However, the existence of multistable behaviour at parameter values away from and before an SNB is also of crucial importance. In particular, they indicate that (i) voltage profile anomalies may occur because of the existence of another attractor, (ii) the destruction of the "other" attractor may result in trajectories which could be interpreted as voltage collapse. In our example, the chaotic attractor itself undergoes a possible global bifurcation and is destroyed. Therefore the stability boundary of the stable operating point must be carefully estimated for parameter values before an SNB.

While static bifurcations are responsible for changing the number of equilibrium points, dynamic bifurcations, such as a subcritical Hopf, are indicative of stability region shrinkage. In two dimensions, the unstable limit cycle, from the subcritical Hopf, forms the exact stability boundary. For dynamics in $n$ dimensions, $n \geqslant 3$, one cannot find any constructive results of this sort unless one can show the existence of quasi-periodic solutions cmbedded in an $(n-1)$-dimensional invariant torus. These higher co-dimension bifurcations are not considered to be sufficiently robust to perturbations in parameter. Several examples can be found in Guckenheimer (1983) where the notion of persistent (robust) bifurcations is elaborated. Under certain very restrictive conditions (Venkatasubramanian et al 1992), the stable manifold of this unstable limit cycle emerging as a result of the subcritical Hopf bifurcation forms part of the exact stability boundary.

The organization of the paper is as follows. In $\S 2$, we review some bifurcation terminology and suggest many possibilities of local dynamical phenomena leading to scenarios of global bifurcation. In $\S 3$, simulation results of one swing equation with load dynamics are presented. Separatc studies (Dobson \& Chiang 1989; Abed et al 1992; Chiang et al 1992) have looked at the same model. The global asymptotic picture of even this rather simple system is far from complete. Only local bifurcations near equilibria or periodic orbits are well understood.

On the other hand, large-scale, classical models, with lossless transmission lines, have relatively simple dynamics. Because they possess energy functions, there are no limit cycles, and trajectories either converge to equilibrium points or are unbounded. The existence of unbounded trajectories will depend on whether the states corresponding to the machine angles are measured $(0,2 \pi)$ or $(-\infty,+\infty)$. Moreover, analytic results of the boundary of the stability regions are well known, even though this boundary is sometimes hard to obtain numerically and may even have a truncated fractal structure (Dobson \& Delchamps 1989; Varghese et al 1993).

The results for classical power systems also hold for structure preserving systems which account for algebraic constraints modelling transmission network load flows as well as flux decay dynamics. Energy functions have been shown to exist, although the construction of these functions is slightly more involved (Tsolas et al 1985). Among local bifurcations only the saddle-node is possible for either the classical or the structure preserving model.

The non-existence of an energy function, and hence the possibility of more complicated dynamics, may have many causes. Lossy transmission lines can prevent the existence of a Lyapunov function (Narasimhamurthi 1984; Chiang 1989). The addition of exciter systems and load dynamics to the swing equations, however, is probably the primary cause of the existence of local dynamic phenomena such as the Hopf bifurcation (Abed \& Varaiya 1984; Rajagopalan et al 1989) 
Load characteristics are known to have a significant effect on system dynamics. Classical load models such as the constant $P Q$, constant impedance, and constant current models are not appropriate to capture voltage dynamics. In $\S 3$, the load is modelled as a differential equation representing the load voltage and angle dynamics. In $\S 4$ we discuss the case where the load model is an algebraic equation. The algebraic equation can be seen as a limiting case of the differential equation model when the load dynamics are varying much faster than the generator dynamics. The state of the system is now constrained to lie on a manifold defined by this algebraic equation. Certain parts of the edges (singularity surfaces) of this manifold may play a role in the analysis of the stability boundary. Citing the example of structure preserving systems, we note that the mere presence of algebraic constraints is not indicative of oscillatory or even more complicated behaviour. An effort to identify certain "critical terms" in the load dynamics for the example in $\S 3$ could be useful in understanding the connection between the shape of the constraint manifold and the onset of solutions that are bounded but do not converge to equilibrium points.

\section{Some bifurcation terminology}

The mathematical literature on bifurcation theory is immensely rich and diverse. A large number of definitions and concepts have been developed to deal with the qualitative behaviour of the orbit structure of parametrized families of autonomous dynamical systems. The purpose of this section is to present some of the basic concepts so as to make this paper fairly self-contained. A fairly detailed survey of bifurcations in power systems that are relevant to voltage collapse, oscillatory and complicated behaviour is given in Varaiya et al (1990). Bifurcation refers to a qualitative change in system trajectories of a parametrized family of dynamical systems as one or more parameters are varied.

Bifurcations in power systems can generally be classified into static and dynamic bifurcations. A static bifurcation occurs when the number of solutions to the real power equations (swing dynamics) changes under parameter variation. For lossless transmission lines, the vector field for power system dynamics is the gradient of some potential energy function $V$. The set on which the gradient $D_{x} V$ is zero forms the set of equilibrium points. The catastrophe set is defined to be points where the Hessian $D_{x x} V$ is rank deficient. Catastrophe theory provides a classification scheme for polynomial approximations of $V$ as given by Thom (1975). Depending on the polynomial approximation, one can predict the static bifurcation set. General power system dynamics do not possess such structure and catastrophe theory results cannot. be applied. However, static bifurcation prediction can be made easier by dimension reduction methods like the Lyapunov-Schmidt technique. The typical static bifurcation in power systems is the saddle-node bifurcation (SNB) when a stable equilibrium and a saddle coalesce to form an unstable equilibrium at which the Jacobian matrix is singular, i.e. it has a zero eigenvalue. The resulting local trajectory (the centre manifold), if oriented towards the direction of decreasing voltage, is interpreted as voltage collapse (Dobson \& Chiang 1989). In the case of differential-algebraic systems, the behaviour of the global extension to the centre manifold will depend on the topological properties of the constraint manifold.

The typical saddle-node bifurcation for the system $\dot{x}=f(x, \lambda)$ is given by $f=0$ and $\operatorname{det}\left(D_{x} f\right)=0$, where $x \in R^{n}$ and $\lambda \in R$. The bifurcation point $\left(x^{*}\left(\lambda^{*}\right), \lambda^{*}\right)$ can be found 
by solving these $n+1$ nonlinear equations. Certain transversality conditions such as $\zeta \times D_{x x} f\left(x^{*}, \lambda^{*}\right) \neq 0$ and $\zeta \times D_{\lambda x} f\left(x^{*}, \lambda^{*}\right) \neq 0$ also need to be satisfied, where $\zeta$ is the left eigenvector corresponding to the zero eigenvalue of $D_{x} f\left(x^{*}, \lambda^{*}\right)$. If $f$ can be derived as the gradient of some potential function, then the catastrophe theory classifies such a bifurcation as a fold catastrophe. Even for $n=2$ the swing dynamics of lossless transmission lines were shown by Arapostathis et al (1982) to have both fold and cusp catastrophes, the latter occurring when three equilibrium points come together. Co-dimension one bifurcations occur on $(n-1)$-dimensional hypersurfaces and split the state space of the system into open sets. Larger co-dimension implies hypersurfaces of dimension less than or equal to $n-2$.

A dynamic bifurcation in power systems occurs when a periodic orbit emerges or disappears. It may take on the form of a Hopf bifurcation, cyclic fold bifurcation, or period doubling bifurcation. A Hopf bifurcation is characterized by the emergence of a periodic orbit (limit cycle) around an equilibrium point. The bifurcation is said to be subcritical (respectively, supercritical) if an unstable (respectively, a stable) periodic orbit emerges around a stable (respectively, an unstable) equilibrium point. A cyclic fold bifurcation occurs when a stable limit cycle collides with an unstable one, and both disappear as the system parameter is further varied. Thus a cyclic fold bifurcation is simply a saddle-node bifurcation of periodic solutions. A period doubling bifurcation refers to the emergence of a stable periodic orbit $C_{2}$ around another periodic orbit $C_{1}$, where the period of $C_{2}$ is approximately twice that of $C_{1}$. The orbit $C_{1}$ is stable before the bifurcation, but loses its stability after the bifurcation. Thus the attracting set of a suitably chosen Poincare first return map, which consists of a single point before a period doubling bifurcation, bifurcates into two points at the bifurcation.

A Hopf bifurcation takes place when the number of the equilibrium points are preserved, but the Jacobian matrix $D_{x} f$ evaluated at an equilibrium point admits a pair of purely imaginary eigenvalues. The normal form for the Hopf bifurcation theorem is strictly defined for two-dimensional systems, but dimension reduction is usually accomplished by using centre manifolds or singular perturbation techniques, as in Abed (1985).

Transversality conditions are imposed to ensure non-degeneracy of the Hopf bifurcation at the place where the pair of eigenvalues cross the $j \omega$ axis. If the pair recrosses the $j \omega$ axis for a neighbouring parameter value, one can classify the bifurcation as non-persistent or non-robust. Degeneracy occurs when the crossing back occurs in a non-transverse fashion. We state these mainly to emphasize that bifurcations that are not persistent may not be observed unless the parameter is quasi-statically varied in a very stow manner. On the other hand, Chen \& Varaiya (1988) show that several degenerate bifurcations are possible for a realistic ninestate power system model. Though analytic results are not easily forthcoming because of the difficulty in calculating the Lyapunov-Schmidt reduction for the nine-state system, it was possible to show correspondences between the normal forms for degenerate bifurcations and numerical results.

Since local bifurcations are easily testable using analytic methods, it is useful to describe some global bifurcations that are a direct consequence of local bifurcations. Of greater significance is the role played by unstable limit cycles, which are not observable in practice; their contribution to the transition of local to global phenomena occurs by their encounters with other limit sets. What follows is a list of such bifurcations. 
(i) Saddle connection: Even for a single generator connected to an infinite bus, which can be regarded as a damped, nonlinear pendulum with a constant forcing in $R^{2}$, it is possible to "balance" the forcing and the damping term to obtain a trajectory that originates from one saddle and terminates in another. The saddle connection signifies a global bifurcation.

For a model with exciter dynamics, a subcritical Hopf bifurcation produces an unstable limit cycle. Under parameter change this limit cycle can grow till it becomes a saddle connection; this is also called a homoclinic orbit.

(ii) Blue sky catastrophe; Two separate subcritical Hopf bifurcations occur in $R^{3}$ with $\lambda \in R^{2}$. The pair of unstable limit cycles evolve in magnitude, under parameter variation, till they meet at a saddle equilibrium point. The two unstable periodic orbits then annihilate each other. This is a global event. The co-dimension of the bifurcation is more than one; so the robustness of the event with respect to parameter perturbation needs further analysis. Before a mutual destruction of the two unstable limit cycles, a homoclinic orbit is formed with the accompanying possibility of complicated transient behaviour. This case is discussed in detail by Wang \& Abed (1992).

(iii) Cyclic fold bifurcation: This is a bifurcation that is very important in the power system example in $\S 3$. An unstable limit cycle emerging from a subcritical Hopf bifurcation coalesces with an existing stable limit cycle, and the pair disappears. Clearly, this sequence, in reverse, is also allowed and is observed in the example in $\S 3$. We note that the shrinkage of the stability domain of the stable operating point commences with the appearance of a pair of unstable-stable limit cycles. One can also classify this as a saddle-node bifurcation in the corresponding discrete time system; the set of difference equations can be arrived at by using a Poincare return map on a transverse hyperplane of dimension $R^{\mathrm{n}-1}$, where the continuous time system state is defined as $x \in R^{n}$. In most cases, obtaining such a "closed form" expression for a Poincaré return map is non-trivial and can only be calculated by the perturbation of integrable trajectories in the continuous time system.

(iv) The Smale horseshoe: In the classical power system model mentioned in $\S 1$, it is possible to obtain complicated trajectories, provided the machine angles are measured in $(0,2 \pi)$. The correct model for the state space of a power system is still a matter of some debate because such a measurement of machine angles does not distinguish a rotating solution from an oscillating one; the former denotes loss of synchronicity of generators and therefore cannot be truly classified as a periodic orbit. Suppose a saddle connection in the machine dynamics of a single swing equation in $(0,2 \pi) \times R$ is perturbed by a periodic solution from another part of the power system. Local analysis near the saddle connection is sufficient to calculate the possibility of the existence of a complicated invariant set that arises from homoclinic tangles and is known as the Smale horseshoe. Indeed, if the periodic forcing perturbation term breaks up the saddle connection in a way that leads to a transversal heteroclinic intersection, then there is a global bifurcation into chaos. A technique for determining if a transversal intersection does occur is known as the Melnikov integral. Details of this technique are given by Kopell \& Washburn (1982) and Salam et al (1984). The Melnikov method is one of the few cases where analytic prediction of global behaviour is possible. Furthermore, one needs to define a trapping region around the homoclinic tangles. Otherwise, trajectories executed on such a set, which is invariant, but not attracting, will not be observable.

(v) Local predictions for period doubling: The study by Alexander (1.986) of a pair 
of generators connected to a lossy line shows that in the case of a supercritical Hopf bifurcation, the stability of the periodic orbit is lost soon afterwards. The normal form for the Hopf bifurcation shows a propensity for the "exchange" of stability similar to the unfolding of the pitchfork. When the stability of the periodic orbit is lost, anothei periodic orbit of twice its period becomes stable. This pattern continues and is known as the period doubling route to chaos. Local testing is good enough for the prediction of each period doubling occurrence. Open questions, like the number of such tests needed before one can safely conclude that the period doubling sequence results in a chaotic limit set, still remain.

\section{Simulation results for a power system model}

In this section, a bifurcation analysis of a three-bus power system model previously proposed (Dobson \& Chiang 1989) is presented. The bifurcation diagrams show that the system exhibits both static and dynamic bifurcations. Furthermore, there are cascades of period doubling bifurcations which lead to chaos. We also study the effects of these bifurcations on system trajectories by investigating the behaviour of system trajectories near the stable operating point for parameter values corresponding to the various types of bifurcation.

\subsection{System model}

The system consists of a load bus and two generator buses. One of the generator buses is treated as a slack bus (infinite bus). The load is modelled as a dynamic induction motor (representing an industrial load) in parallel with a constant $P Q$ load and a constant impedance load (representing residential and commercial loads). We follow the same notation as Abed et al (1992). This gives rise to a system of four differential equations given by

$$
\begin{aligned}
& \dot{\theta}=\omega, \\
& M \dot{\omega}=-d_{m} \omega+5 V \sin (\delta-\theta+0 \cdot 0873)+0 \cdot 564, \\
& \varepsilon \dot{\delta}=-a_{0} V^{2}-a_{1} V+\left[Q_{e}(\delta, V)-Q\right], \\
& \varepsilon b_{0} b_{1} \dot{V}=a_{0} b_{2} V^{2}+\left(b_{2} a_{1}-\varepsilon b_{0}\right) V+\varepsilon\left[P_{e}(\delta, V)-P\right]-b_{2}\left[Q_{\mathfrak{e}}(\delta, V)-Q\right] .
\end{aligned}
$$

The variables $\theta$ and $\omega$ are the machine angle (in radians) and angular velocity (in radians per second). $V$ and $\delta$ represent the voltage magnitude (in p.u.) and angle of the load bus. The machine damping coefficient is $d_{m}$, and $M$ is the mass of the generator. The coefficients $a_{i}$ and $b_{i}$ are derived from an induction motor load. The constant $s$ is small so that the $(V, \delta)$ dynamics are faster than that of $(\theta, \omega)$. The premultiplication by $\varepsilon$ of (3) shows that $\delta$ is the fast variable within the load subsystem (3)-(4). The terms in square brackets are the load flow equations for a constant $P Q$ load, where $P$ and $Q$ are the real and reactive power demand at the load bus, respectively. The electrical real power $P_{e}(\delta, V)$ and reactive power $Q_{e}(\delta, V)$ can be expressed as

$$
\begin{aligned}
& P_{e}(\delta, V)=-\gamma_{1} V \sin \left(\delta+\psi_{1}\right)-\gamma_{2} V \sin \left(\delta-\theta+\psi_{2}\right)+\gamma_{3} V^{2}, \\
& Q_{e}(\delta, V)=+\gamma_{1} V \cos \left(\delta+\psi_{1}\right)+\gamma_{2} V \cos \left(\delta-\theta+\psi_{2}\right)-\gamma_{4} V^{2},
\end{aligned}
$$


where $\gamma_{1}$ are products of conductances and constant voltages behind reactance and $\psi_{i}$ are constant phase shifts. After substituting (5)-(6) into (2)-(4) the following structure appears in the load dynamics:

$$
\begin{aligned}
& \dot{\delta}=\alpha_{0} V^{2}-\alpha_{1} V \cos \left(\delta-\theta-\phi_{1}\right)-\alpha_{2} V \cos \left(\delta-\phi_{2}\right)-\alpha_{3} V+\alpha_{4} Q+\alpha_{5}, \quad(7) \\
& \dot{V}=-\beta_{0} V^{2}+\beta_{1} V \cos \left(\delta-\theta-\phi_{3}\right)+\beta_{2} V \cos \left(\delta-\phi_{4}\right)+\beta_{3} V-\beta_{4} Q-\beta_{5}
\end{aligned}
$$

where $\alpha_{i}, \beta_{i}$, and $\phi_{i}$ are constants given by $\alpha_{0}=496.87, \alpha_{1}=166.66, \alpha_{2}=666.66$, $\alpha_{3}=93.33, \alpha_{4}=33.33, \alpha_{5}=43.33, \beta_{0}=78.76, \beta_{1}=26.21, \beta_{2}=104.86, \beta_{3}=14.52$, $\beta_{4}=5.22, \beta_{5}=7.03, \phi_{1}=0.0873, \phi_{2}=0.2094, \phi_{3}=0.0124, \phi_{4}=0.1346$.

\subsection{Bifurcation analysis}

Three sets of bifurcation diagrams were drawn for different machine damping coefficients given by $d_{m}$. The parameter that was varied in each of the bifurcation diagrams is $Q$, the reactive power demand. The bifurcation diagram that exhibits the richest qualitative behaviour, including two period-doubling routes to chaos, is for the values $d_{m}=0.05$ and $M=0.3$. With $M=0.3$ fixed, as $d_{m}$ is increased to 0.1 , all period-doubling bifurcations are suppressed. At $d_{m}=0.114$ the only bifurcation is the SNB. For $d_{m}=0.05$ we observe six types of bifurcations.

(i) $\mathrm{CFB}$, cyclic fold bifurcation: $Q=10 \cdot 81813$,

(ii) $\mathrm{PDB}_{1}$, period doubling bifurcation: $Q=10 \cdot 87327$,

(iii) UHB, unstable or subcritical Hopf bifurcation: $Q=10.94681$,

(iv) $\mathrm{PDB}_{2}$, period doubling bifurcation: $Q=11.38779$,

(v) SHB, stable or supercritical Hopf bifurcation: $Q=11.40665$,

(vi) SNB, saddle node bifurcation: $Q=11 \cdot 41136$,

We first present simulation results for $d_{m}=0 \cdot 05$. Figures $1 \mathrm{a}$ and $\mathrm{b}$ are local bifurcation diagrams of load voltage $V$ versus $Q$ and load angle $\delta$ versus $Q$. For the range of $Q$ values considered, the system has two equilibrium points (stationary points, operating points). One of them is always unstable. In fact, the Jacobian at this equilibrium has one real and positive eigenvalue and three open left half-plane eigenvalues, so it is a type-1 unstable equilibrium. The location of this unstable equilibrium is indicated by the lower branch in the $V-Q$ plot and the upper branch in the $\delta-Q$ plot. Denote the other equilibrium by $x_{s}$. At $Q=10.94681$, there is a UHB with the emergence of an unstable limit cycle around $x_{s}$ for $Q<Q_{\mathrm{UHB}}$, hence by the exchange of stability formula described by Abed \& Varaiya (1984), $x_{s}$ loses stability for $Q>Q_{\mathrm{JHB}^{\circ}}$ At $Q=11.40665$, there is an $\mathrm{SHB}$, so $x_{s}$ regains stability for $Q>Q_{\text {SHB }}$. At $Q=11.41136$ an SNB occurs and the equilibria coalesce and become a single equilibrium at which the Jacobian is singular. Local analysis shows that there is a pair of complex eigenvalues and two real and negative eigenvalues associated with the Jacobian at $x_{s}$. Figure $2 \mathrm{a}$ shows the real and imaginary parts of the pair of complex eigenvalues, with the arrow indicating their movement as $Q$ is increased from 10.5. The eigenvalues cross the $j \omega$ axis transversely at $Q=Q_{\mathrm{UHB}}$ so that $x_{s}$ becomes unstable for $Q>Q_{\mathrm{UHB}}$, and recross it at $Q=Q_{\mathrm{SHB}}$ so that $x_{s}$ regains stability for $Q>Q_{\mathrm{SHB}}$. For $Q$ near $Q_{\mathrm{SNB}}$, the real part of the eigenvalue is very sensitive to changes in $Q$. At $Q=Q_{\mathrm{SNB}}$, one of the two real and negative eigenvalues crosses the $j \omega$ axis and becomes positive. This reactive power demand corresponds to the system 

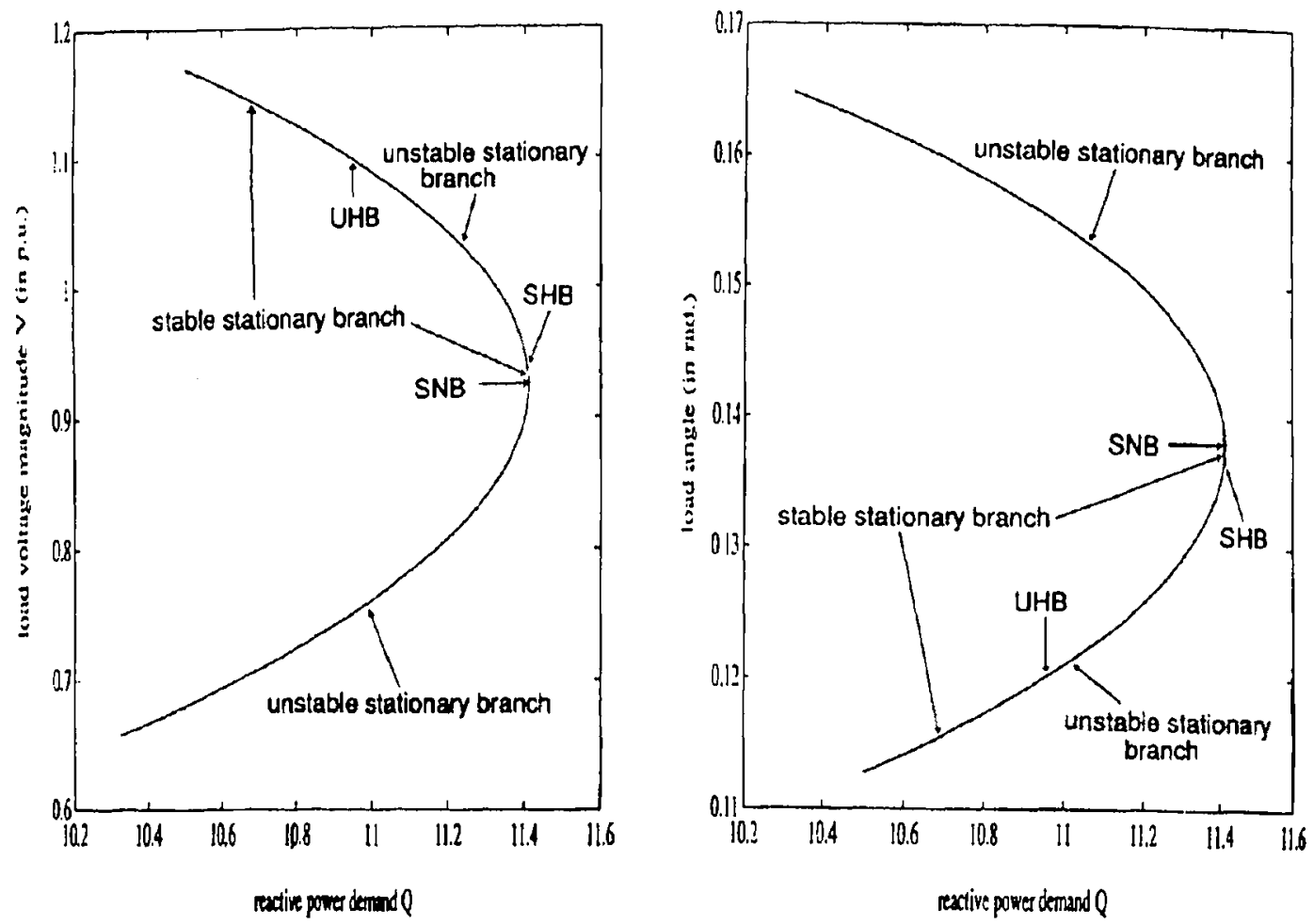

Figure 1. Voltage magnitude (a) and load angle (b), of the two equilibria, damping $=0.05$.

steady-state operating limit, and the system has no operating solution for $Q>Q_{\mathrm{SNB}}$. Figure $2 b$ gives a summary of the stability nature of the two equilibrium points.

Whereas figures $1 \mathrm{a} \& \mathrm{~b}$ only show the stationary branches (location of equilibria), figure 3 also shows the maximum amplitude of the periodic branches. From figure $1 \mathrm{a}$, we know that a UHB occurs at $Q=10.94681$. Figure 3 shows the maximum amplitude of the unstable limit cycle emerging from this UHB for $Q<Q_{\mathrm{UHB}}$. On decreasing $Q$ to 10.81813 , this oscillation is destroyed in a CFB, where collision with a stable limit cycle occurs. If we follow the stable periodic branch, there is a period doubling bifurcation at $Q=Q_{\mathrm{PDB}_{1}}=10.87327$. We refer to the calculation (Alexander 1986) that a period doubling bifurcation signals the appearance of a stable limit cycle of twice the period of the stable periodic branch. Moreover, as indicated in figure 3, the stable periodic branch becomes unstable for $Q>Q_{\mathrm{PDB}_{1}}$. Figure 3 also shows an SHB at $Q=11.40665$. If $Q$ is decreased the stable oscillation emerging from the SHB will undergo a period doubling bifurcation at $Q=Q_{\mathrm{PDB}_{2}}=11.38779$, so the stable oscillation from SHB becomes unstable for $Q<Q_{\mathrm{PDB}_{2}}$. Numerical computations show that the unstable branch coming from $\mathrm{PDB}_{2}$ coincides with that coming from $\mathrm{PDB}_{1}$.

Figure 4 shows both the maximum and minimum amplitudes of oscillation. For example, at $Q=11.2$, the unstable oscillation has a maximum voltage of 1.0628 p.u. and a minimum voltage of 0.8354 p.u. We note that for $Q=10.85582<Q_{\mathrm{PDB}_{1}}$ in the $V$ versus $Q$ plot as shown in figure 4 , the stable equilibrium $x_{s}$ is surrounded by a stable limit cycle $C_{s}$ and an unstable limit cycle $C_{u}$. Figure 5 shows the unstable limit cycle in the $(\theta, V)$ space. Figure 6 shows the time waveform for the voltage component of the unstable oscillation. The point $(0.707,0 \cdot 0,0 \cdot 1179,0.99)$ on $C_{\mu}$ is used as an initial condition to get these plots. Figure 7 shows the stable limit cycle in $(\theta, V)$ space, while 
(a)
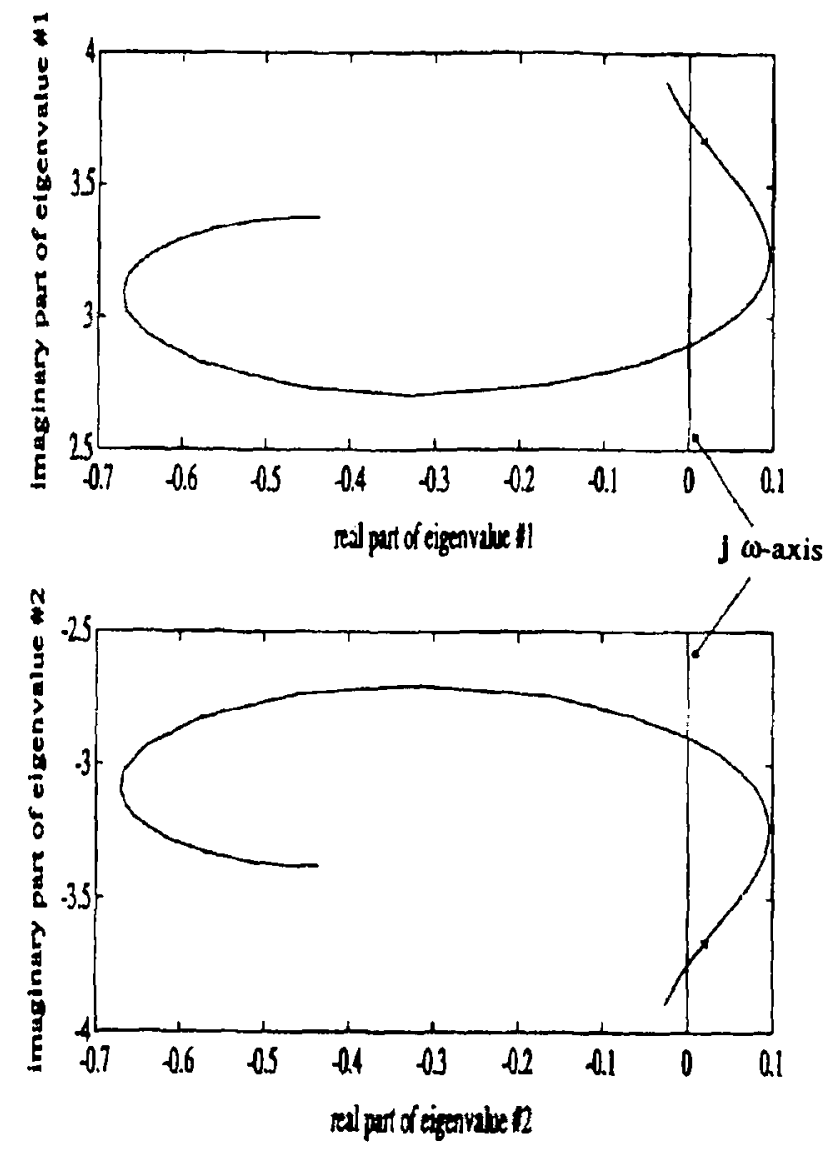

(b) Equilibrium number $1\left(x_{s}\right)$ :
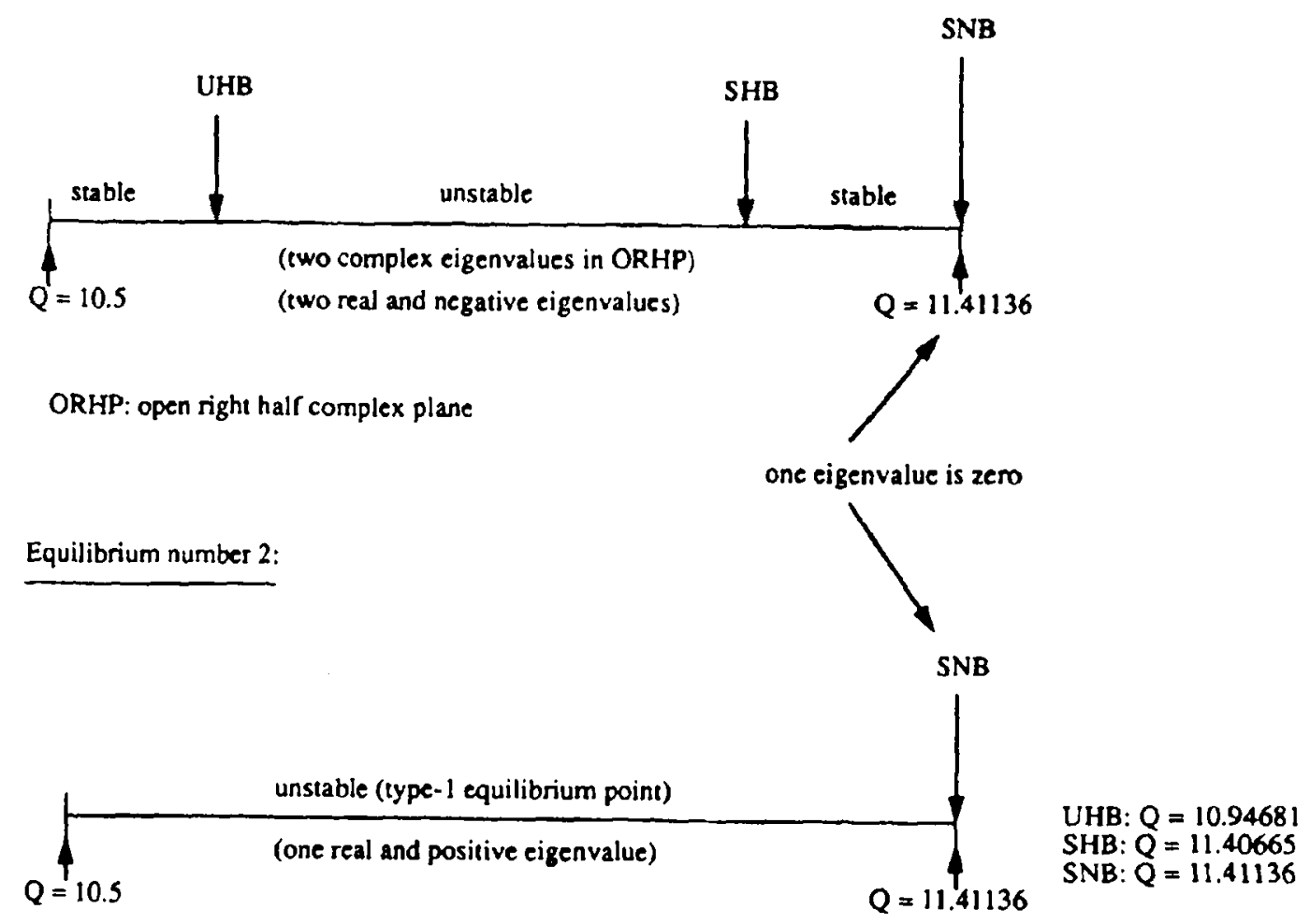

Figure 2. (a) Location of the complex eigenvalues as $Q$ varies. (b) Stability of equilibrium points showing local bifurcation values. 


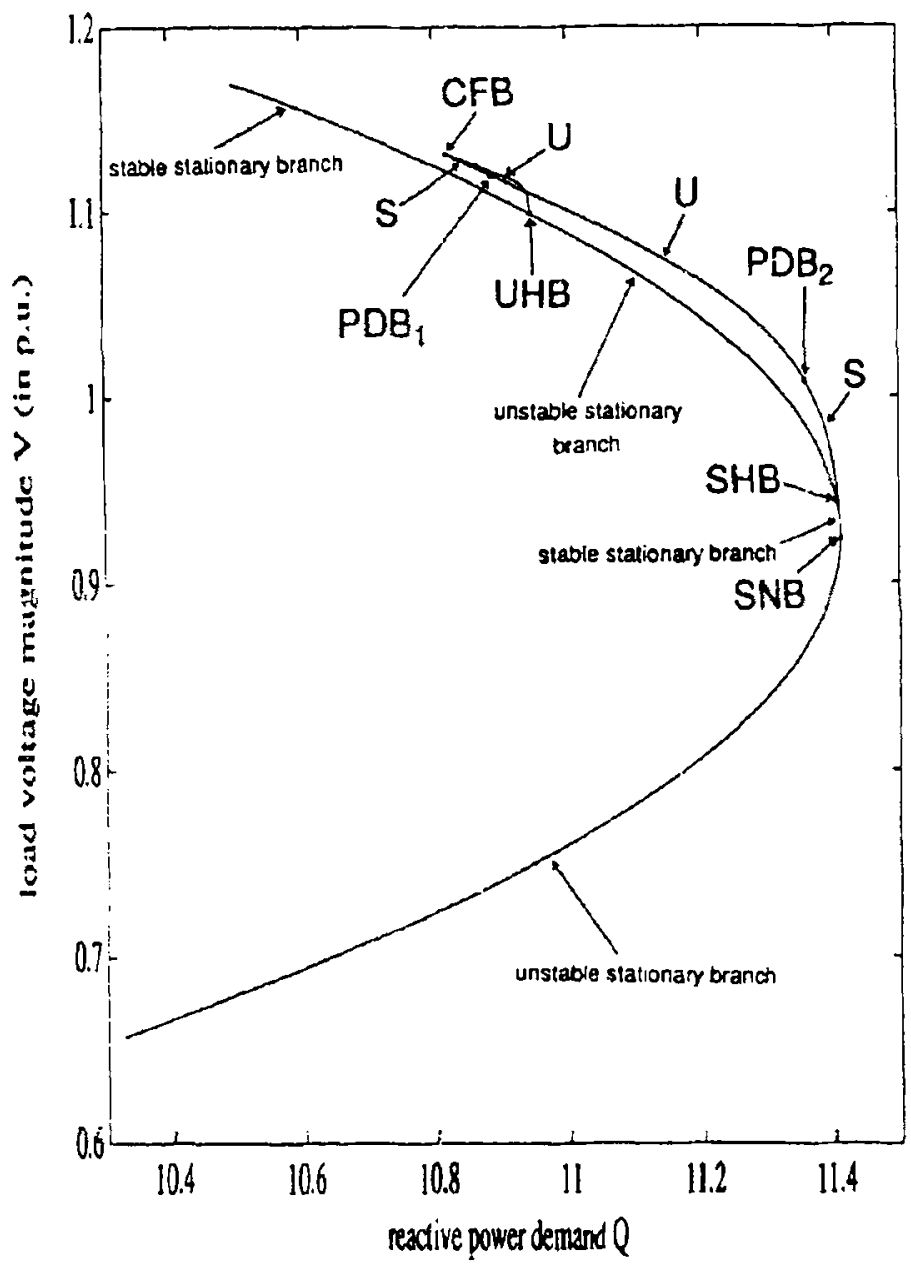

S: stable periodic branch

U: unstable periodic branch
Figure 3. Bifurcation diagram for $V$, damping $=0.05$. The maximum amplitudes of the periodic branches are shown.

figure 8 is the corresponding time waveform for its voltage component. The initial condition used is $(0 \cdot 735,0 \cdot 0,0 \cdot 1179,0 \cdot 99)$. From figure 4 (and simulation data) the maximum voltage amplitudes of $C_{s}$ and $C_{u}$ are $1 \cdot 1256$ p.u. and 1.1266 p.u., respectively. Also the minimum voltage amplitudes of $C_{s}$ and $C_{u}$ are 0.8517 p.u. and 0.9686 p.u., respectively. So neither the $V$ component of $C_{s}$ nor that of $C_{u}$ surrounds the other. However the situation is quite different in other variables. For example, in the $\theta$ variable, $C_{s}$ encompasses $C_{u}$. Hence, in the $\theta$ component $C_{u}$ acts as a separatrix between the domain of attraction of the stable equilibrium $x_{3}$ and that of the stable limit cycle $C_{s}$. Figures 9 and 10 show plots of $\theta$ versus time for the unstable and stable oscillations, respectively. We point out that for $Q$ values near 10.85582 , the system exhibits bistable behaviour in which the stable equilibrium $x_{s}$ is surrounded by a stable limit cycle. The question of estimating the size of the domain of attraction of $x_{s}$ is therefore crucial since a disturbance can easily lead to oscillatory behaviour if the domain of attraction is small. This is an important transient stability problem. We will have more discussion on the domain of attraction of $x_{s}$ in $\$ 3.4$.

Figures 11 and 12 show bifurcation diagrams for larger values of machine damping: $d_{m}$ equals $0 \cdot 1$ and $0 \cdot 114$. From the system equations (1)-(4) we note that different values of $d_{m}$ have no effects on the locations of the two equilibrium points. However, the Jacobian will be different. In figure 11 there is no PDB. Also implied is the absence of chaos, since the route to chaos (for the system being considered here) is followed via a sequence of period doubling bifurcations. We will discuss this in greater detail later when we examine the chaotic nature of the system trajectories for some values 


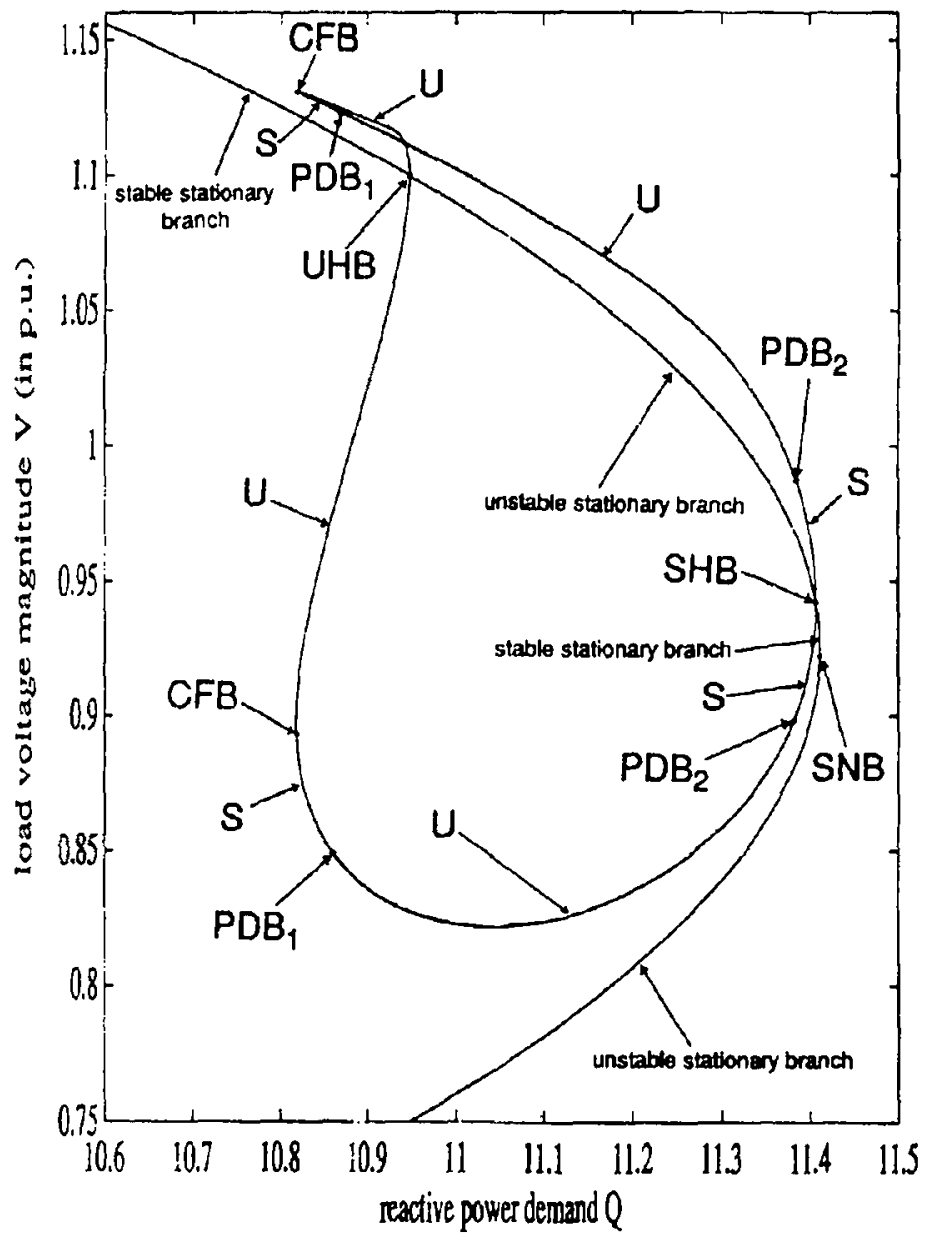

S: stable periodic branch

$U:$ unstable periodic branch

Figure 4. Bifurcation diagram for $V$, damping $=0.05$. Both maximum and minimum amplitudes of the periodic branches are shown.

of $Q$. It may be possible to show local results similar to those of Alexander (1986) that calculate the value of $d_{m}$ at which no period doubling bifurcation can occur. Figure 11 also shows that a UHB occurs at $Q=11.3365$ and an SHB occurs at $Q=11.3975$. The unstable and stable limit cycles emerging from these two bifurcations collide at a CFB at $Q=11 \cdot 3295$. Hence the distance between the two values $Q_{\mathrm{UHB}}$ and $Q_{\text {SHB }}$ for $d_{m}=0.1$ is closer than that for $d_{m}=0.05$. In figure 12 , all the dynamic bifurcations CFB, UHB and SHB disappear. There is only a static bifurcation SNB at $Q=11.41136$. In fact, as $d_{m}$ is increased, UHB and SHB will come closer until they coincide with each other and disappear at some value $Q=Q_{\mathrm{HB}}$. This corresponds to a degenerate Hopf bifurcation and an eigenvalue plot similar to that in figure 2a shows a non-transversality in the eigenvalue crossing of the jw axis.

\subsection{Chaotic dynamics}

With $d_{m}=0.05$ fixed, numerical results show that there is a cascade of period doubling bifurcations starting at $Q_{\mathrm{PDB}_{1}}=10.87327$. For $Q=10.87857$, a $V$ versus $\theta$ plot is shown in figure 13. A double periodic loop is clearly observed. Figure 14 is the corresponding plot of $V$ versus time and shows that the "lower envelope" exhibits modulation between the two values 0.83 and 0.855 . Further period doubling bifurcations occur as $Q$ is increased. These period doublings accumulate in a dense fashion on the attractor shown in figure 15 for $Q=10.894$. The "erratic" nature of the plot of $V$ versus time is shown in figure 16. Lyapunov exponents, defined by 


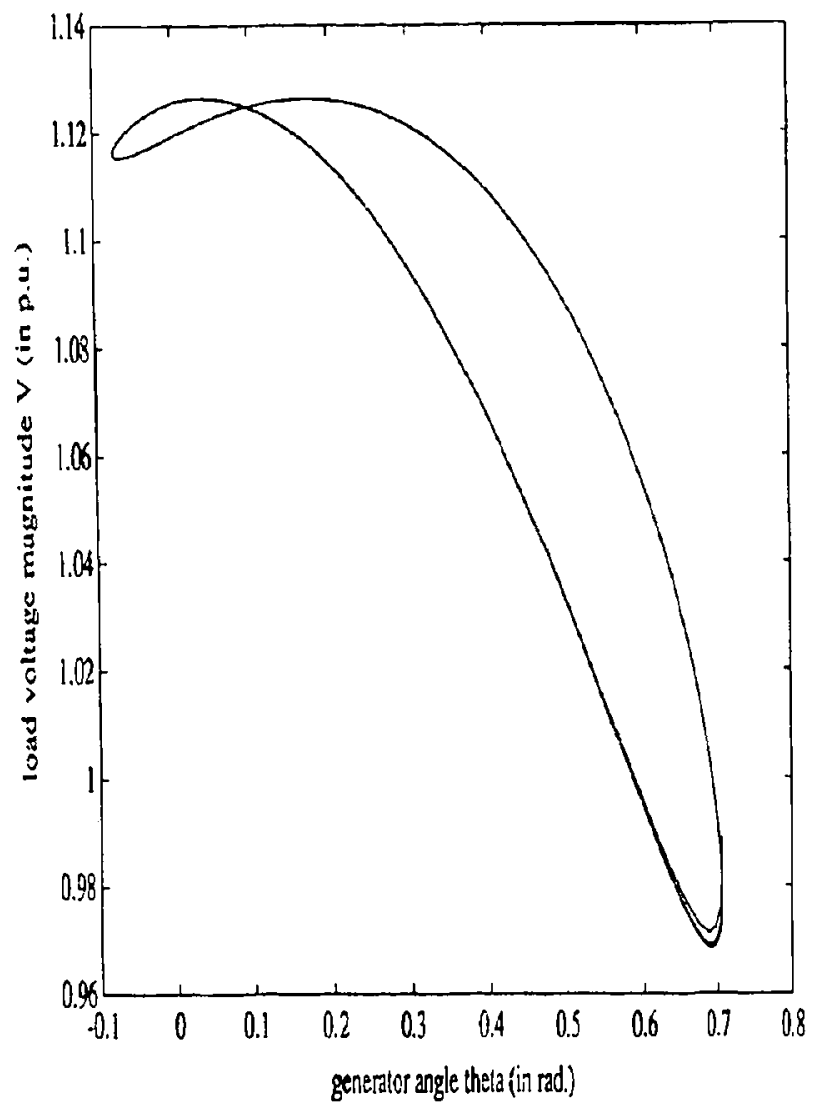

Figure 5. Trajectory showing the unstable limit cycle, $Q=10 \cdot 85582$.

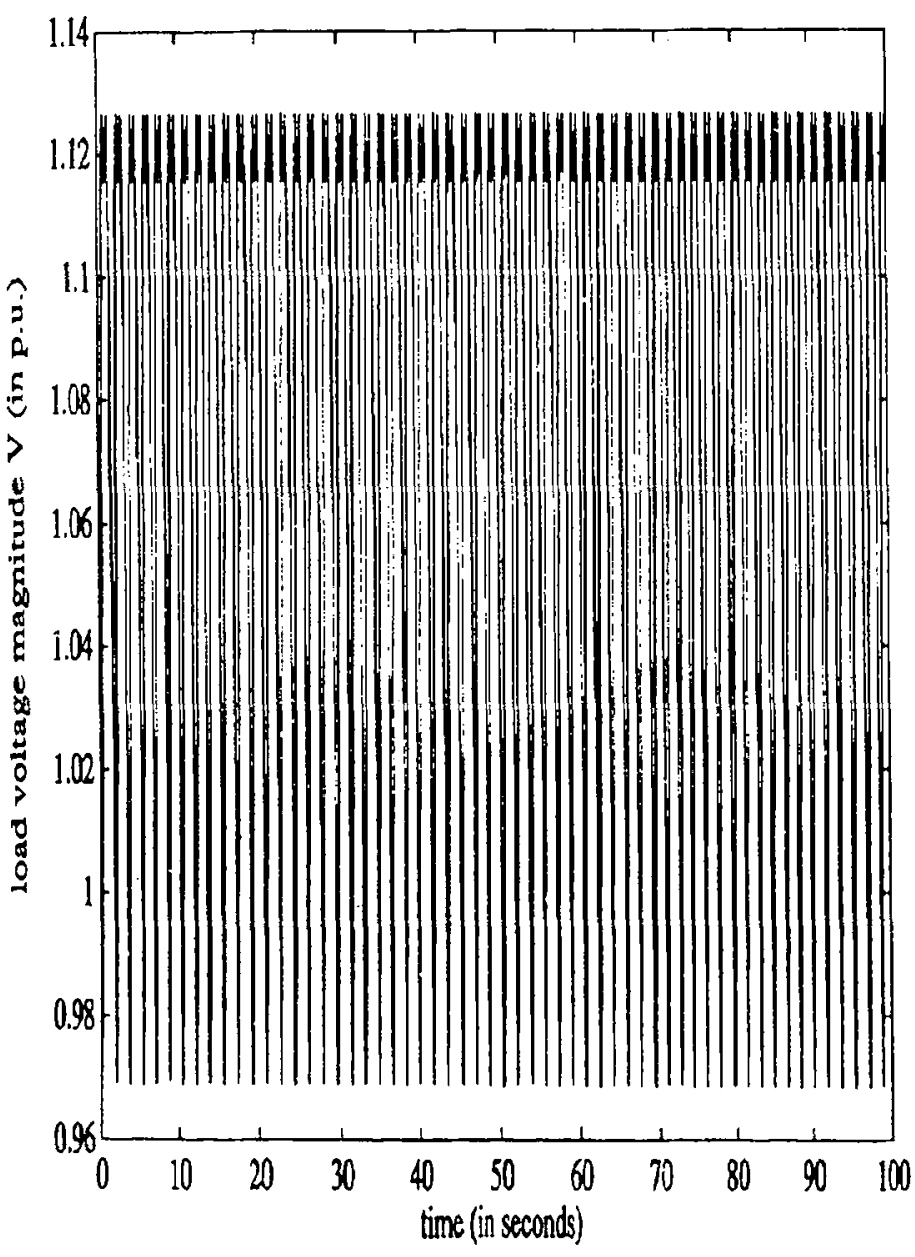

Figure 6. Time waveform of the unstable limit cycle, $Q=$ 10.85582 

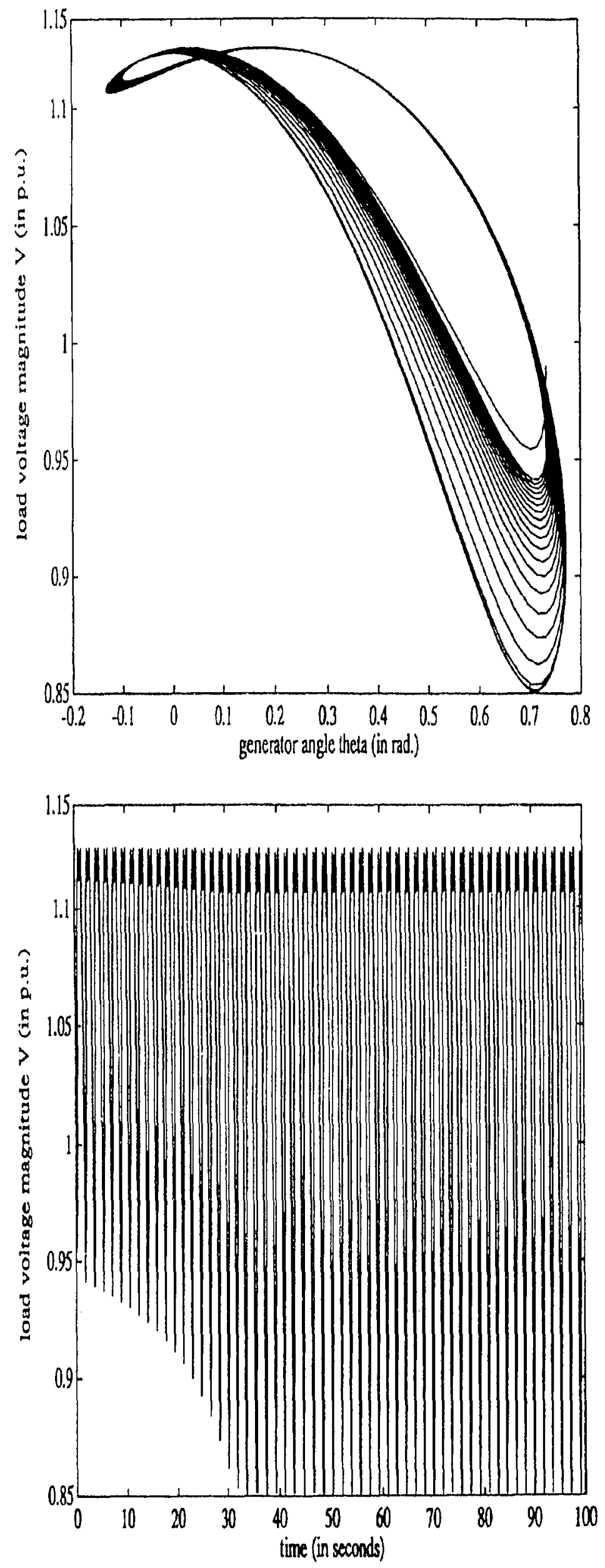

Figure 7. Trajectory attracted to a stable limit cycle, $Q=$ 10.85582 .
Figure 8. Time waveform of the stable limit cycle, $Q=$ 10.85582 . 

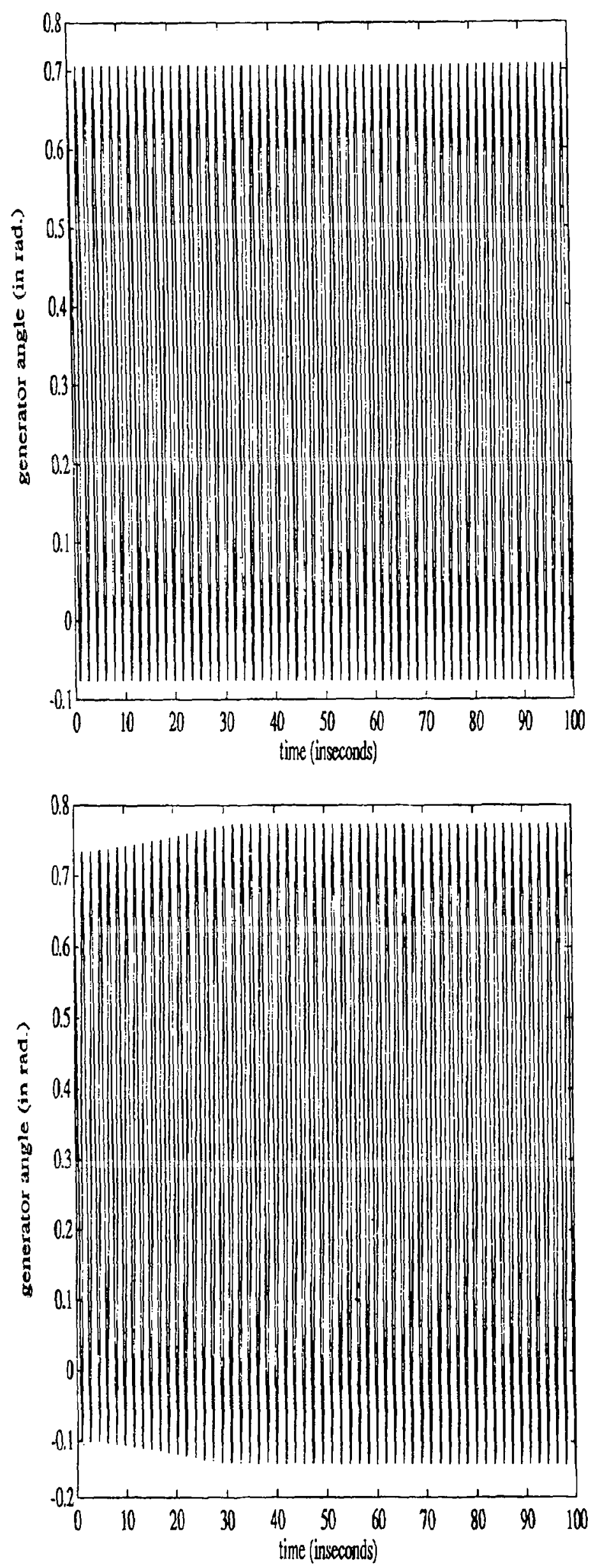

Figure 9. Time waveform of the unstable limit cycle, $Q=$ 10-85582.

Figure 10. Time waveform of a stable limit cycle, $Q=10.85582$. 


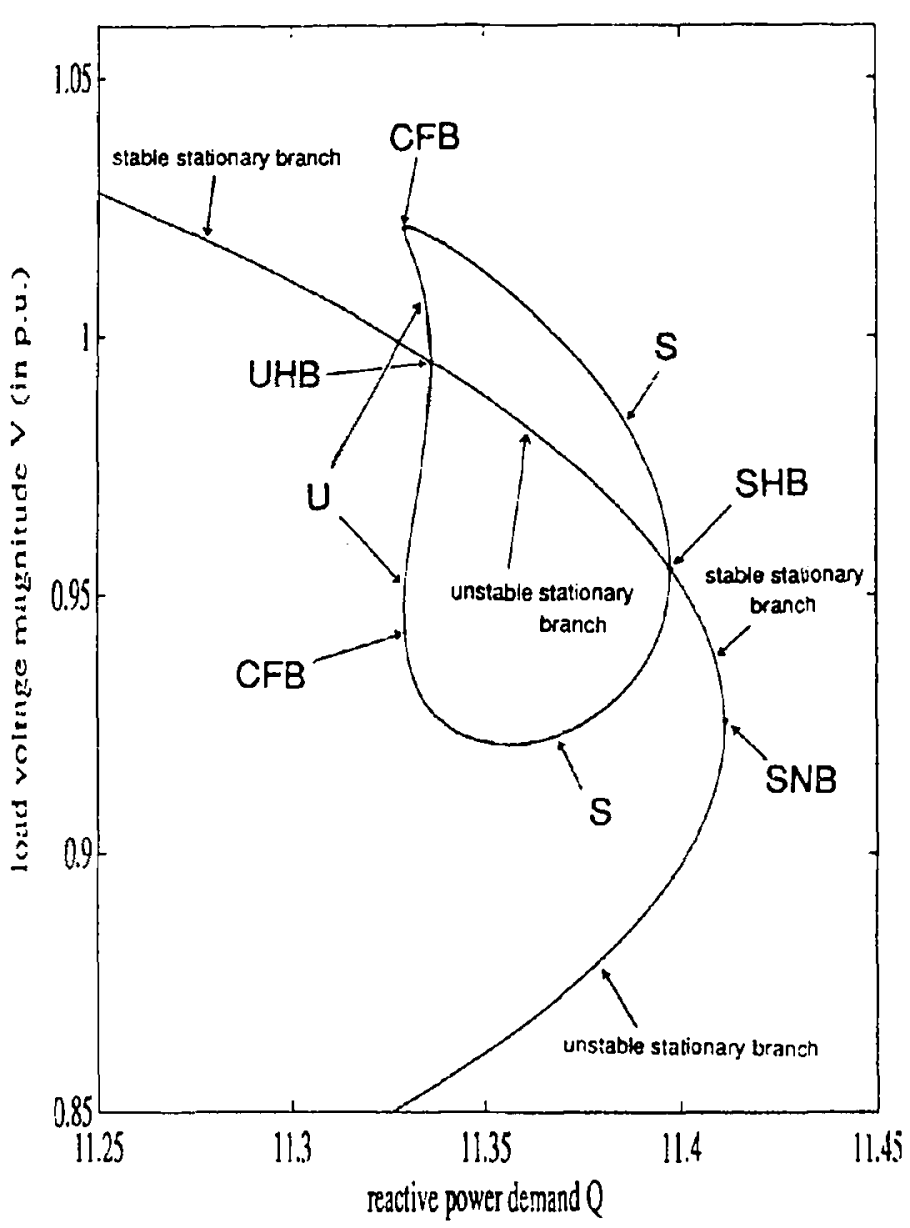

S: stable periodic branch

$\mathrm{U}$ : unstable periodic branch
Figure 11. Bifurcation diagram for $V$, damping $=0 \cdot 1$.

Guckenheimer \& Holmes (1986), which measure the average exponential stretching of initially nearby points can be used to confirm that the system exhibits chaotic trajectories for this value of $Q$. This chaotic structure is first observed at about $Q=10 \cdot 89$, but disappears when the reactive power demand is increased to $Q=10.894$. We do not yet know how the strange attractor gets annihilated. Several possibilities are detailed by Grebogi et al (1983) for the Henon attractor.

When $Q$ is decreased beyond $\mathrm{PDB}_{2}$, there is a similar cascade of period doubling bifurcations. Similar to the other chaotic attractor that exists for $10 \cdot 89 \leqslant Q \leqslant 10.894$, this attractor exists for $Q$ values in the range $(11 \cdot 37,11 \cdot 38)$. It is interesting to note that the strange attractor at $Q=10.894$ is larger in size than the one obtained for $Q=11.377$. Figure 17 shows a comparison of the two attractors in the $(\theta, V)$ space. Finally, figure 18 shows the partition of the parameter space, $10.5 \leqslant Q \leqslant 11.41136=$ $Q_{\mathrm{SNB}}$, with respect to the various bifurcation values described in this section.

\subsection{Domain of attraction of $x_{s}$}

Suppose we want to characterize the set of all initial conditions that converge to the stable operating point $x_{s}$ for different values of $Q$, with $d_{m}$ equal to 0.05. Denote such an open set by $A\left(x_{s} ; Q\right)$. A' meaningful range of $Q$ for such an investigation would be for $Q \leqslant Q_{\mathrm{UHB}}=10.94681$. Since $x_{s}$ loses stability for $Q>Q_{\mathrm{UHB}}, A\left(x_{s} ; Q\right)$ will shrink to the empty set as $Q \uparrow Q_{\mathrm{UHB}}$. However, before this event takes place, the size and shape of $A\left(x_{s} ; Q\right)$ will undergo some interesting qualitative changes as a result of other bifurcations that occur for $Q<Q_{\mathrm{UHB}}$. First we note that for $Q<Q_{\mathrm{CFB}}=10.81813$, 

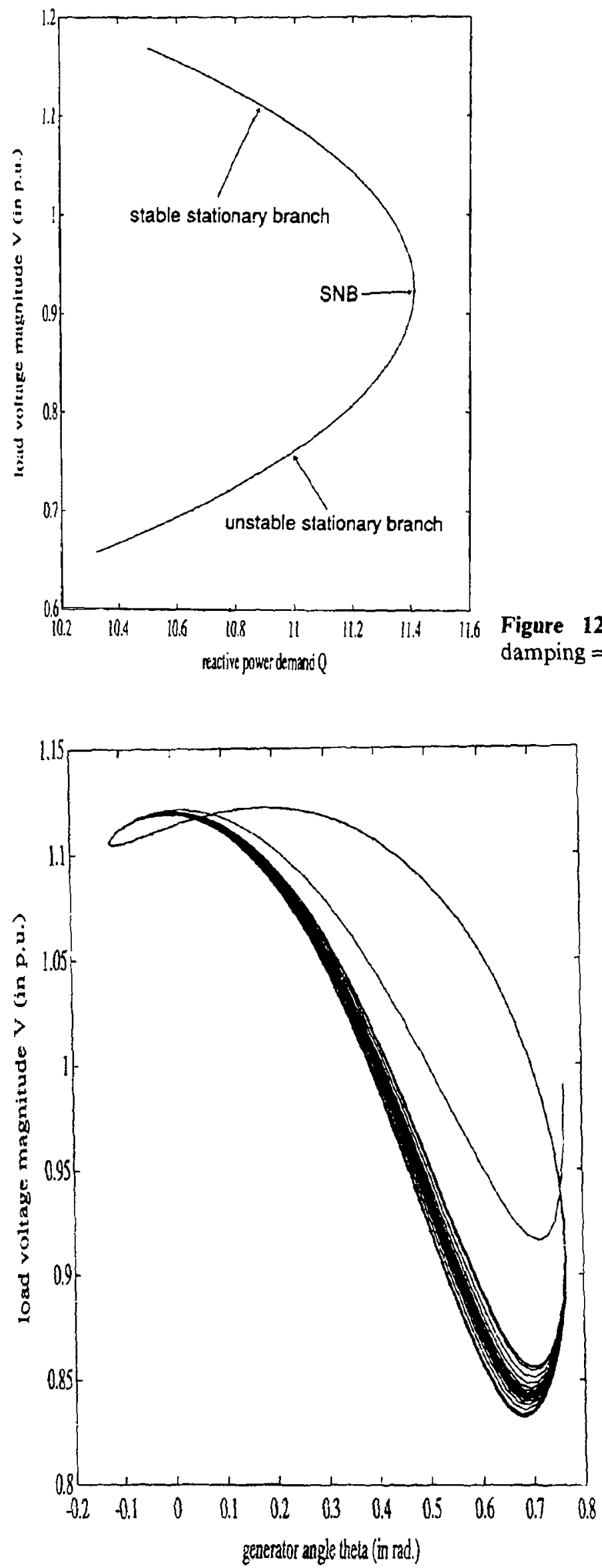

Figure 12. Bifurcation diagram for $V$, damping $=0.114$.
Figure 13. A double-loop periodic solution, $Q=10.87857$. 

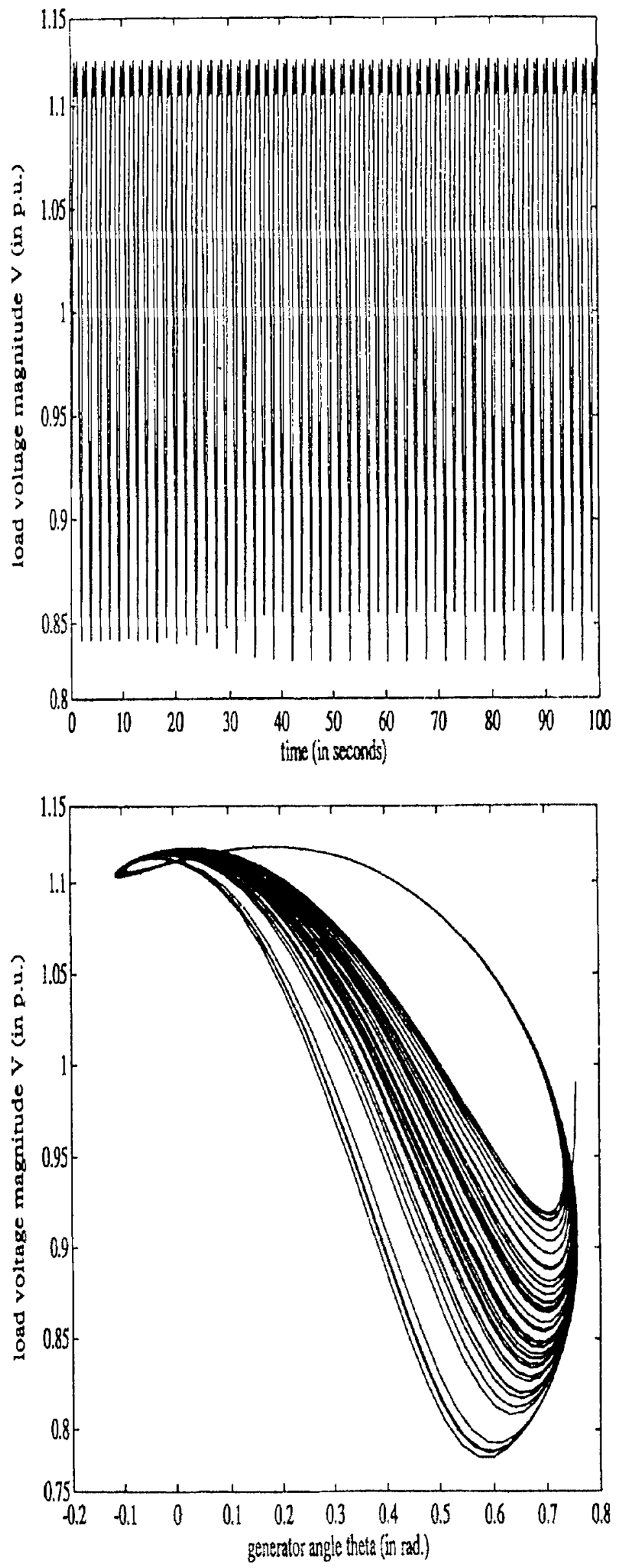

Figure 14. A double-loop periodic solution, $Q=10.87857$.

Figure 15. Chaotic dynamics due to successive $\mathrm{PDB}, Q=$ 10.894 . 

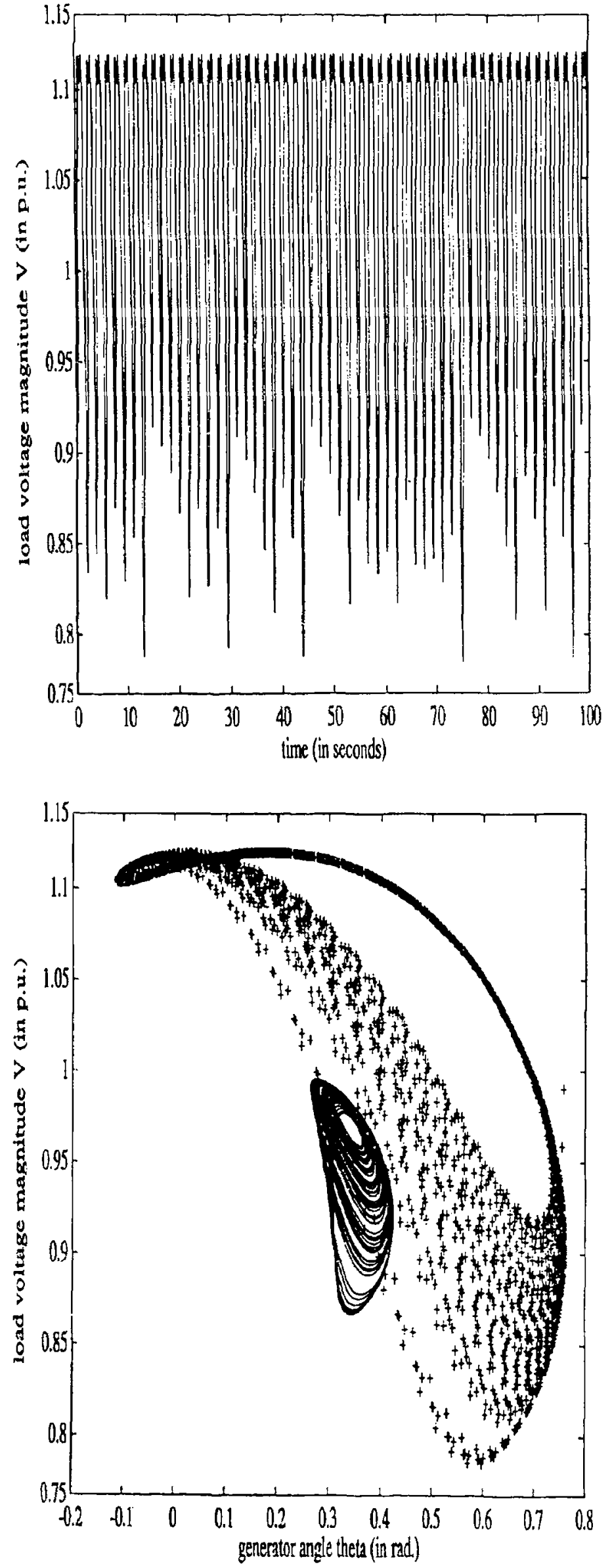

Figure 16. Chaotic dynamics due to successive PDB, $Q=$ 10.894 .

Figure 17. Two chaotic attractors; $(+) Q=10 \cdot 894,(-) Q=$ 11.377. 


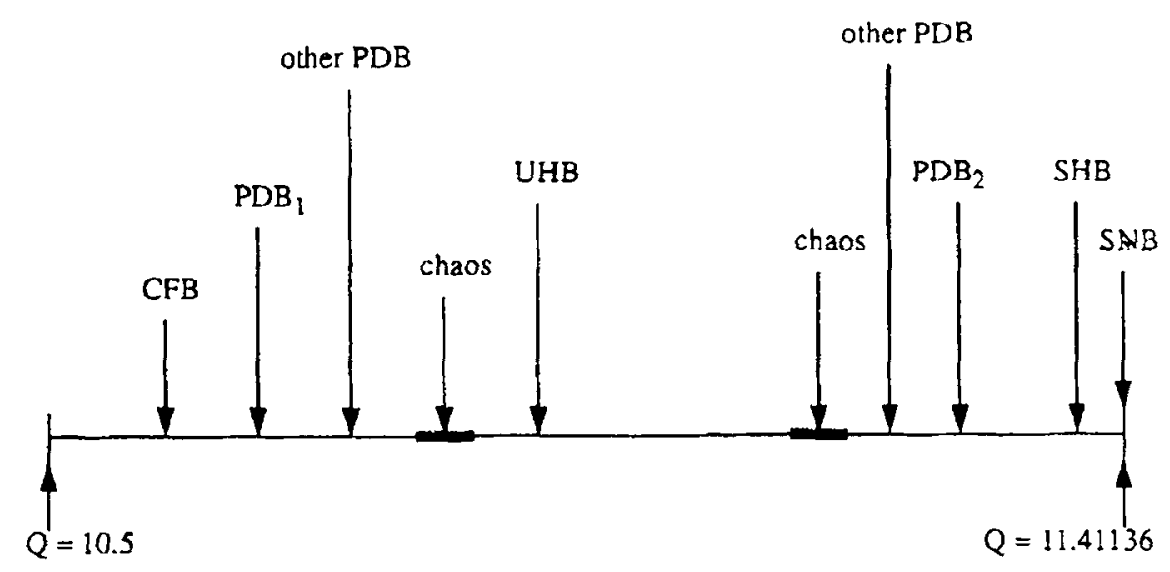

CFB: $Q=10.81813, P_{1} B_{1}: Q=10.87327$, UHB: $Q=10.94681$, chaos: $10.89 \leqslant Q \leqslant 10.894$ SNB: $Q=11.41136$, SHB: $Q=11.40665, P_{2}: Q=11.38779$, chaos: $11.37 \leqslant Q \leqslant 11.38$

Figure 18. Partition of the parameter space showing local and global bifurcation values.

the state space has only two invariant sets - the type- 1 equilibrium and $x_{s}$ - so the stability region $A\left(x_{s} ; Q\right)$ can be really large. In fact, it may be unbounded. As soon as $Q$ reaches $Q_{\mathrm{CFB}}$, the emergence of a limit cycle around $x_{s}$ will suddenly put a constraint on the size and shape of the domain of attraction. Since $A\left(x_{s} ; Q\right)$ represents the set of points a power engineer would like the system to operate at, it is important to find out the types of qualitative changes in $A\left(x_{s} ; Q\right)$ as $Q \uparrow Q_{\text {CFB }}$.

The composition of $\partial A\left(x_{s} ; Q\right)$ (the boundary of $A\left(x_{s} ; Q\right)$ ) as given in Chiang et al (1988) and Zaborsky et al (1988) is the union of the closure of the stable manifolds of the type-1 unstable equilibrium points on $\partial A\left(x_{s} ; Q\right)$. Type-1 implies that the unstable equilibrium point has a one-dimensional unstable direction. If a type- 1 equilibrium point is on the boundary, then some component of its unstable manifold will necessarily converge to the stable equilibrium $x_{s}$. In what follows we will only consider this component of the unstable manifold of the type-1 equilibrium.

From our numerical results we find some apparent contradictions. For some values of $Q$ less than $Q_{\mathrm{UHB}}=10.94681$ but greater than $Q=10.894$ at which chaos gets annihilated, the unstable manifold of the type-1 equilibrium does not converge to $x_{s}$. Figure 19 shows that for $Q=10.945$, the voltage component of the unstable manifold oscillates around and away from the stable equilibrium $x_{s}$, and later experiences a sudden sharp drop (voltage collapse). So the type-1 equilibrium is not on $\partial A\left(x_{s} ; Q\right)$. The theory by Chiang et al (1988) and Zaborski et al (1988) requires the existence of an energy function, and since there is no such function for a model that allows the presence of oscillations, the plot in figure 19 does not contradict the theory. However, the result implies that if the system initially operates at a point near $x_{s}$ for $Q=10.945$, it may experience voltage collapse without ever converging to $x_{s}$. Once again, this brings up the important issue of estimating the size of $A\left(x_{s} ; Q\right)$ and investigating the components on $\partial A\left(x_{s} ; Q\right)$. For $Q=11.378$ the system exhibits chaotic behaviour and both equilibrium points are unstable (see figure $2 \mathrm{~b}$ ). Figure 20 shows that the voltage component of the unstable manifold of the type-1 equilibrium converges to the chaotic attractor. Some details of unstable limit sets on $\partial A\left(x_{s}\right)$ for systems without energy functions are summarized in $\S 5$. 


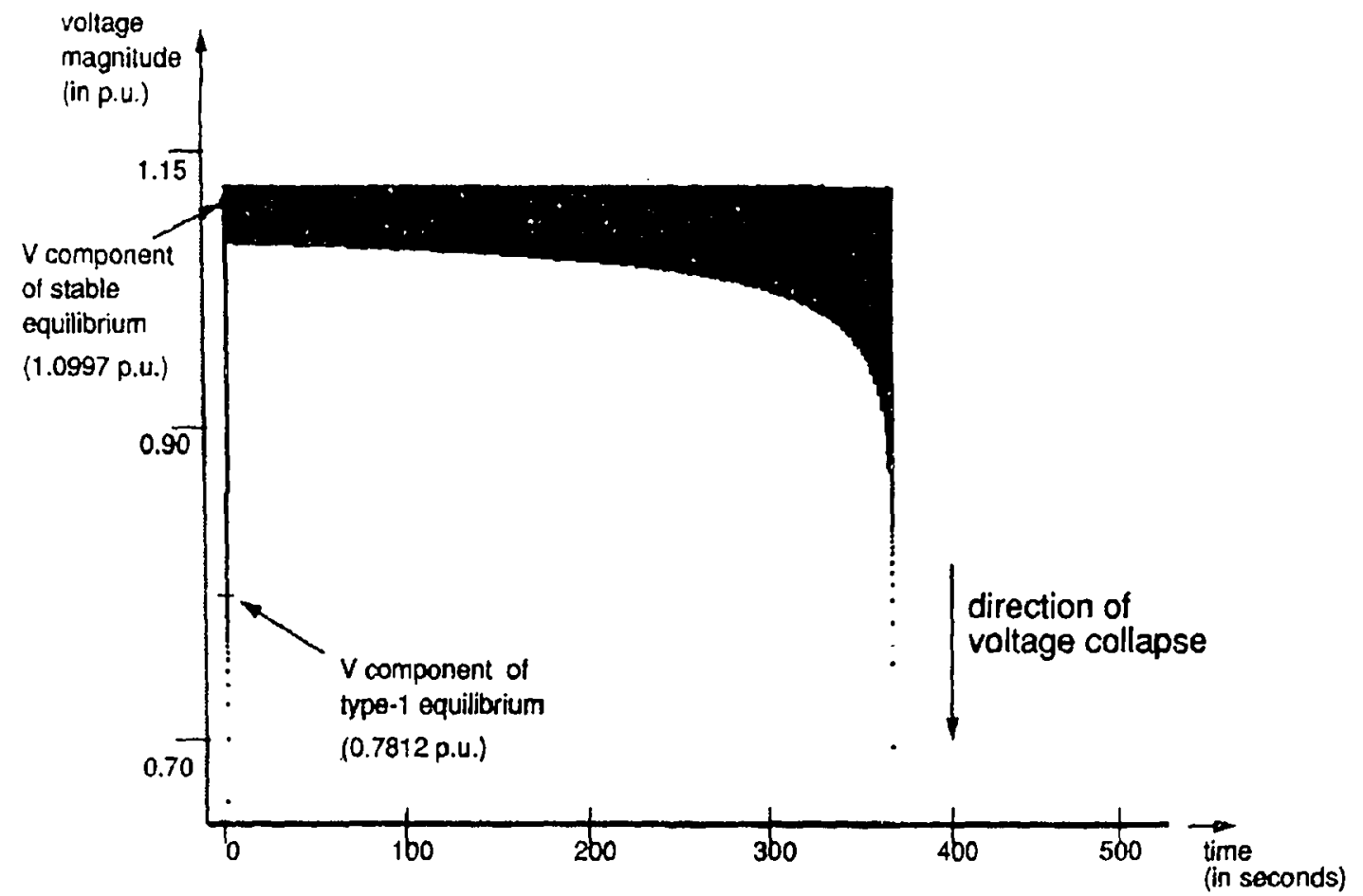

Figure 19. Unstable manifold of type-1 equilibrium point (voltage collapse) $\left(Q=10.945<Q_{\text {UHB }}\right)$.

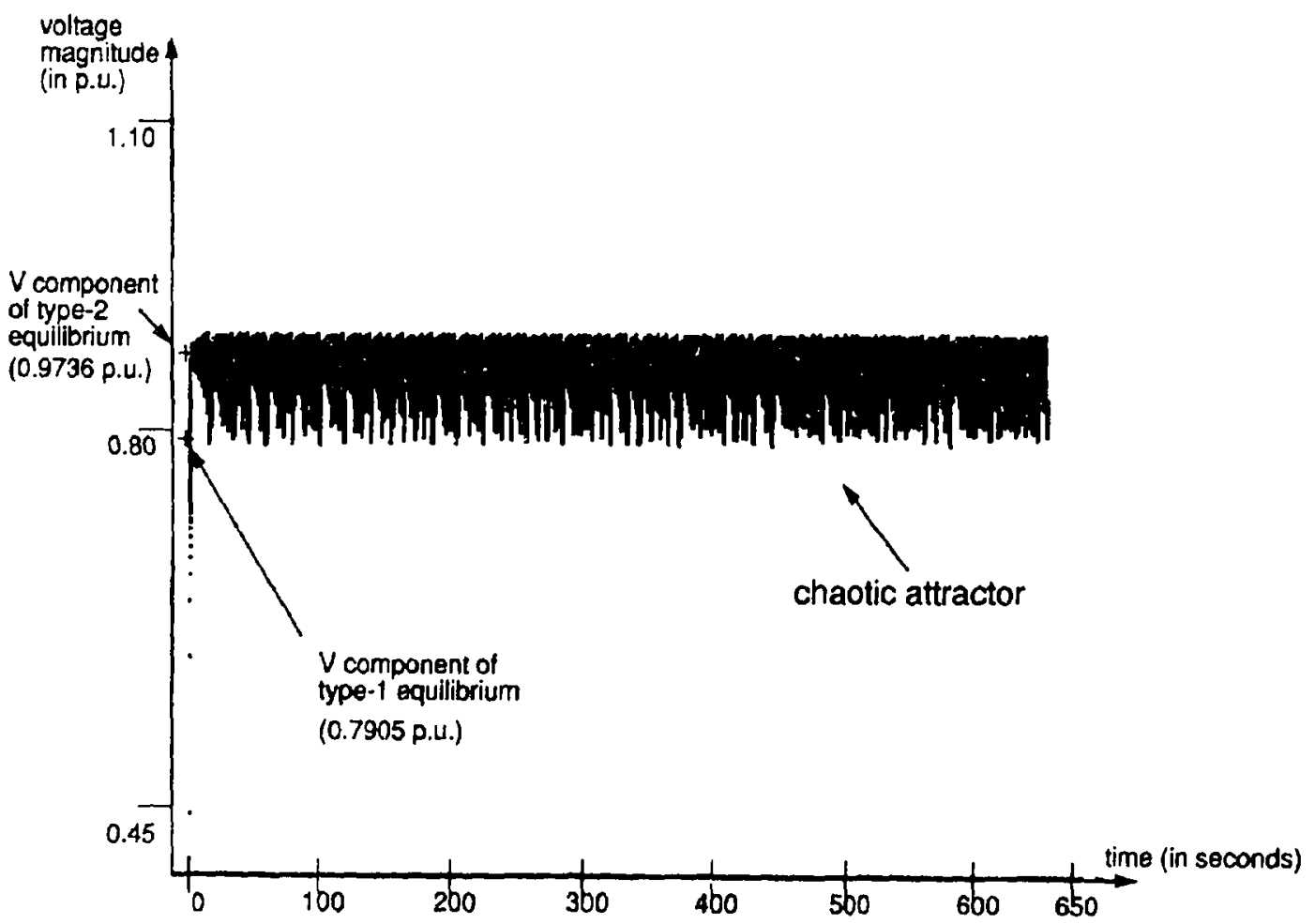

Figure 20. Unstable manifold of type-1 equilibrium point attracted to the chaotic attractor $(Q=11 \cdot 378)$. 


\section{Algebraic constraints}

In this section we present some classifications of differential algebraic models of power systems. The model is general enough so that energy functions, which have strictly negative time derivatives along non-trivial trajectories, may not exist. Consider a differential algebraic equation (DAE) defined by

$$
\begin{aligned}
& \dot{x}=f(x, y), \\
& 0=g(x, y),
\end{aligned}
$$

where $x \in R^{n}, y \in R^{m}$, and $m<n$. The functions $f, g$ are assumed to be $C^{r}$ smooth, $r \geqslant 2$. For brevity, we write $f \equiv f(x, y), g \equiv y(x, y)$. Typically the $x$ variable represents the machine dynamics as well as flux decay and the $y$ variable represents the load flow constraints and the flow of power in the transmission network.

Define the two sets

$$
\begin{aligned}
& L:=\{(x, y): g(x, y)=0\}, \\
& S:=\left\{(x, y) \in L: \operatorname{Det}\left(D_{y} g\right)=0\right\} .
\end{aligned}
$$

The set $L$ is an $n$-dimensional embedded submanifold determined by the algebraic constraint $g(x, y)=0$ and is called the constrained manifold. The set $S$ is called the singular surface or singularity set. It is necessary that all valid solutions lie on $L$; the basic idea being that if the rank condition $\operatorname{det}\left(D_{y} g\right) \neq 0$ (equivalently, $\operatorname{rank}\left(D_{y} g\right)=m$ ) in the implicit function theorem is satisfied, then (10) is used for solving $y$ in terms of $x$ and substituting in (9). Thus $y$ varies $C^{r}$ smoothly as a function of $x$ on the set $L \backslash S$. By Sard's theorem (Milnor 1963), regular points are dense in $L$. This implies that even when $\operatorname{det}\left(D_{y} g\right)=0$, the $m \times(m+n)$ matrix $\left(D_{x} g \mid D_{y} g\right)$, with $m<n$, has rank $m$ almost everywhere. These assumptions are needed to weed out "bad" sets in $S$.

The singular surface $S$ splits $L$ into open sets labeled $\left\{L_{k}\right\}$. A component $L_{k}$ is considered stable (respectively, unstable) if all the eigenvalues of $D_{y} g$ computed at any point in $L_{k}$ have negative (respectively, positive) real parts. Although we use similar notations, we do not adhere to the assumption that all $L_{\mathbf{k}}$ are stable as in Venkatasubramanian et al (1992). The significance of unstable $L_{k}$, even for systems with energy functions, is illustrated below.

Results in Takens (1976) state that if the vector field for (9)-(10) is $C^{r}$ smooth, $r \geqslant 3$, and if $D_{y} g$ is symmetric, then points where $D_{y} g$ has a single zero eigenvalue is dense in $S$. This non-degenerate rank condition for $D_{y} g$ helps in classifying SNB that occur on $S$. Some descriptions of voltage collapse in structure-preserving systems are postulated by Fekih-Ahmed \& Chiang (1992). For structure preserving systems, the only local bifurcations allowed are SNB. They are split into (a) regular SNB and (b) non-regular SNB. Non-regular SNB belong to the class of singularity induced bifurcations, which refers to the collision of a pair of equilibrium points that are on $S$. For a non-regular SNB, the centre manifold is undefined and typically an eigenvalue will tend to $c 0$.

We assume that $L$ is bounded which in turn implies that each of the open sets $L_{k}$ is bounded. It then follows that for a regular SNB, the global extension to the centre manifold, denoted as $W_{c}^{+}$in positive time, can either converge to a limiting set on a component $L_{k}$ or reach $S$ in finite time. The centre manifold $W_{c}^{+}$cannot be unbounded because the components $\left\{L_{k}\right\}$ are all assumed to be bounded. Could the 
condition that $W_{c}^{+} \rightarrow S$ in finite time be necessary for voltage collapse? It turns out that there is yet another possibility. After $W_{c}^{+}$reaches $S$ there could be a "jump" to a stable member $L_{k}$. This implies "voltage recovery". A jump to an unstable component would mean continued voltage collapse. Such a sequence of jump behaviour has been used to explain several nonlinear circuit phenomena (Sastry \& Desoer 1981; DeMarco \& Bergen 1987).

We point out that because of the high dimensionality of a typical power system, one needs to classify the various behaviours of trajectories when $S$ is encountered. For example, we describe below how certain points in $S$ may have pseudo-equilibrium type behaviour; yet other parts may contribute to finite escape time: In addition, the notion of $W_{c}^{+} \rightarrow S$ may be unrealistic. After a static bifurcation, parameters in the equation $g=0$, such as the reactive power demand, will probably be unable to maintain a constant value; the shape of $L$ will therefore change and the analysis will not be valid. We follow some of the notation from Takens (1976) for a formal approach to the study of implicit differential algebraic equations of the form (9) and (10). A natural way to write the motion on $L$ is to use the implicit function theorem and the chain rule to solve for $\dot{g}=0$. Provided one starts on $g=0$, the trajectory remains on a connected component of $L$. By the chain rule, $\dot{g}=D_{y} g \cdot \dot{y}+D_{x} g \cdot \dot{x}=0$, so (9) and (10) can be rewritten as

$$
\begin{aligned}
& \dot{x}=f, \\
& \dot{y}=-\left(D_{y} g\right)^{-1} D_{x} g . f .
\end{aligned}
$$

One can further simplify by writing $h=\operatorname{Adj}\left(D_{y} g\right) D_{x} g \cdot f$ and $\Delta=\operatorname{det}\left(D_{y} g\right)$ to obtain

$$
\begin{aligned}
& \dot{x}=f, \\
& \dot{y}=-h / \Delta .
\end{aligned}
$$

Using the singular state-dependent time scaling $\mathrm{d} t / \mathrm{d} \tau=\Delta$, we get

$$
\begin{aligned}
& \dot{x}=\Delta . f, \\
& \dot{y}=-h .
\end{aligned}
$$

The time derivatives in (17)-(18) are with respect to the state-dependent time variable $\tau$. Note that if the trajectories of (15)-(16) remain on the component of $L$ where $\Delta>0$, then they are topologically equivalent to the trajectories of (17)-(18). On the other hand, if they stay on the component of $L$ where $\Delta<0$, then they are subject to time reversal with respect to trajectories of (17)-(18). These time reversals endow certain components of $S$ with interesting properties. Consider, for example, the notion of "pass through" trajectories. These are solutions that pass through $S$ at places where (15)-(16) exhibits a $0 / 0$ form. Time reversal effects for $\Delta<0$ transform these "pass through" directions to local stable or unstable manifolds of the pseudo-equilibrium points described below for $S_{1}$.

We split $S$ into 3 subsets. We assume that all these subsets are "nice" in the sense that the sets $S_{i}$ are assumed to be either $n-1$ or $n-2$ dimensional, smooth, embedded submanifolds. Details of showing that the "bad" sets are lower dimensional can be found in the original work of Takens (1976). We briefly describe the subsets as
(a) $S_{1}=\{(x, y) \in S: h=0\}$,
(b) $S_{2}=\left\{(x, y) \in S \backslash S_{1}:\left(D_{y} \Delta\right) \cdot h=0\right\}$,
(c) $\mathrm{S}_{3}=\left\{(x, y) \in S \backslash\left(S_{1} \cup S_{2}\right)\right\}$. 
In many lower dimensional systems, $S_{1}$ and $S_{2}$ may be empty. However, the stable manifolds consisting of trajectories that converge to elements in $S_{1}$ or $S_{2}$ are also interesting and can turn out to be components of basin boundaries as described below.

(i) The embedded submanifold denoted by $S_{1}$ has a maximal dimension of $n-2 . S_{1}$ is considered to be a pseudo-equilibrium surface because the constraints $h=0$ and $\Delta=0$ zero out the vector field for (17)-(18). For the "nice" components of $S_{1}$, it is further assumed that the rank of $D_{y} g$ is $m-1$ and that the Jacobian of (17)-(18) has only 2 eigenvalues with non-zero real part. In $S_{1}$, only 3 types of local behaviour are possible. Elements of $S_{1}$ behave like pseudo saddles, pseudo sinks or pseudo sources. (ii) $S_{2}$ is called a semi-singular surface because the trajectories are tangential to $S$ at every element in $S_{2}$. An additional inequality constraint given by $D_{y}\left\{\left(D_{y} \Delta\right) h\right\} h \neq 0$ is invoked so that the trajectories do not cross $S$ in a degenerate fashion. $S_{2}$ also has maximal dimension $n-2$. Elements in $S_{2}$ are, similarly, categorized into semi-saddles, semi-sources and semi-sinks.

(iii) $S_{3}$ is the most regular part of $S$. It has a maximal dimension of $n-1$. At most two trajectories either terminate in or originate in elements of the "nice" components of $S_{3}$ with infinite speed. $S_{3}$ is responsible for phenomena such as finite escape time.

As we have noted in $\S 3$, a detailed characterization of $S$ may not be useful in the prediction of voltage collapse using $W_{c}^{+}$. Assuming that the shape of $L$ is invariant after a static bifurcation may not be realistic. On the other hand, we noted in $\S 1$ that stability domain shrinkage was a precursor to SNB. The voltage profile could be affected because of transient instability. In order to motivate a discussion on the effect of $L$ on stability domain shrinkage, we show an example of a single swing equation with a reactive power constraint. This is a modified example from Varaiya et al (1990) and belongs to the class of structure preserving systems. We have set all constants such as the mass of the generator, the damping coefficient and the conductance to unity. The state vector $(\theta, \omega, V)$, represents the machine angle, angular velocity and terminal voltage magnitude. We write

$$
\begin{aligned}
& \dot{\theta}=\omega, \\
& \dot{\omega}=-\omega-V \sin (\theta)+P, \\
& 0=-V^{2}+V \cos (\theta)-Q .
\end{aligned}
$$

Here

$$
\begin{aligned}
& L=\left\{(\theta, \omega, V):-V^{2}+V \cos (\theta)-Q=0\right\}, \\
& S=\{(\theta, \omega, V) \in L:-2 V+\cos (\theta)=0\} .
\end{aligned}
$$

$L$ is a maximal two-dimensional submanifold and $S$ is a maximal one-dimensional submanifold. Define $\theta^{*}:=\cos ^{-1}(2 \sqrt{Q}) . S$ can also be written as $\left[\left( \pm \pi \mp \theta^{*}\right), \omega\right.$, $( \pm \sqrt{Q})]$. It can be calculated that $S_{1}$ is equal to $\left[\left( \pm \pi \mp \theta^{*}\right), 0,( \pm \sqrt{Q})\right]$. Whether the points in $S_{1}$ are pseudo-saddles, pseudo-sinks or pseudo-sources is determined by the Jacobian of the vector field corresponding to (17)-(18). Recall that, the points where at most 2 eigenvalues have nonzero real part are dense in $S$. For this case, $S_{1}$ is somewhat degenerate because $S_{2}$ appears to coincide with $S_{1}$. Closer examination reveals that the non-crossing over condition that is needed for belonging to $S_{2}$ is violated. On the other hand, trajectories approach $S_{1}$ in a tangential manner. We treat $S_{2}$ as empty. $S_{3}=S \backslash S_{1} . S$ splits $L$ into 2 open sets such that

$$
\begin{aligned}
& L \backslash S=\left\{L_{1} \cup L_{2}\right\}, \\
& L_{1}=\{(\theta, \omega, V) \in L: V>[\cos (\theta)] / 2\}, \\
& L_{2}=\{(\theta, \omega, V) \in L: V<[\cos (\theta)] / 2\} .
\end{aligned}
$$


Note that $L_{1}$ is stable, $L_{2}$ is unstable, and the assumption (Venkatasubramanian et al 1992) which requires all components $\left\{L_{k}\right\}$ to be stable is violated for this example.

Consider the $(\theta, \omega)$ system for transient stability study. Suppose $V$ was fixed and the algebraic constraint was absent. The stability boundary only consists of the stable manifold of the type-1 unstable equilibrium point, $x^{\prime \prime}$. Now introduce $L$ and the associated induced dynamics of $V$ or $L$. A component of $S_{3}$, consisting of the line $\theta=\pi-\theta^{*}$, cuts through the (original) stability domain in the $(\theta, \omega)$ plane. The stable manifolds of elements in $S_{1}$ are not considered as candidates for the stability boundary. This is because $S_{1}$ does not have a non-empty intersection with the stability domain. The stability boundary in the $(\theta, \omega)$ plane, therefore, consists of part of the stable manifold of $x^{\mu}$ and part of $S_{3}$. Note that $x^{\mu}$ was determined by soiving for the zeroes of (19) and (20). After including $L$, we have to recalculate $x^{u}$ by also solving for the zeroes of (21); and so $x^{u}$ will have moved a little. Nevertheless, this demonstrates the shrinkage of the stability domain.

\section{Concluding remarks}

In seeking explanations other than saddle-node bifurcation to voltage collapse, we have presented a numerical example in $\S 3$ where the domain of attraction $A\left(x_{s} ; Q\right)$ of the stable operating point undergoes some qualitative changes at the onset of a dynamic bifurcation such as the cycle fold bifurcation at $Q_{\mathrm{CFB}}=10.81813$. As we have discussed in $\S 3$ that for $Q=10.945<Q_{\mathrm{UHB}}$, voltage collapse can take piace and type-1 equilibrium does not lie on $\partial A\left(x_{s} ; Q\right)$. Hence the structures of $A\left(x_{s} ; Q\right)$ and $\partial A\left(x_{s} ; Q\right)$ can be rather complicated. In elaborating on $A\left(x_{s}\right)$ one is led to define the presence of unstable limit sets on the boundary $\partial A\left(x_{s}\right)$. In systems that possess energy functions, these limit sets reduce to unstable equilibrium points. Furthermore only type-1 equilibrium points that have $(n-1)$-dimensional stable manifolds need be considered. This is proved by Tsolas et al (1985). However, as we have seen in $\S 4$, the imposition of algebraic constraints may cause $A\left(x_{s}\right)$ to become smaller even for the structure preserving systems considered (Tsolas et al 1985). For such systems, parts of $S$ and the stable manifolds of the pseudo/semi-equilibrium points can be included in $\partial A\left(x_{s}\right)$. Referring to the three-bus model studied in $\S 3$, we make the following remarks:

(i) There are at most three attractors for any value of $Q \in\left[10 \cdot 5, Q_{\mathrm{SNB}}=11 \cdot 41136\right]$. Denote the 3 attractors as $R_{1}, R_{2}$ and $R_{3}$. We consider infinity $( \pm \infty)$ to be an attractor. Label $\pm \infty$ as $R_{1}$.

(ii) The attractor labelled $R_{2}$ is the one that we are most interested in. For $Q<Q_{\mathrm{UHB}}$ and $Q>Q_{\mathrm{SHB}}$, we find that $R_{2}$ is $x_{s}$, the stable operating point. For values of $Q$ in between these $Q_{\mathrm{UHB}}$ and $Q_{\mathrm{SHB}}, R_{2}$ disappears since $x_{s}$ becomes unstable and the Jacobian has a pair of complex open right half plane eigenvalues.

(iii) The attractor labelled $R_{3}$ makes its appearance at $Q=Q_{\text {CFB }}$ as a stable limit cycle. The attractor $R_{3}$ undergoes several dynamic bifurcations, both local and global in nature, after $Q_{\mathrm{PDB}_{1}}$. The period doubling cascade transforms $R_{3}$ into a chaotic attractor; a possible global bifurcation eliminates $R_{3}$ at a $Q$ value near 10.894. $R_{3}$ reappears just before $Q_{\mathrm{PDB}_{2}}$ as another chaotic attractor. Between $Q_{\mathrm{PDB}_{2}}$ and $Q_{\mathrm{SHB}}$, $R_{3}$ is a stable limit cycle.

(iv) For values of $Q$ in between 10.894 (which corresponds to the disappearance of the first chaotic attractor) and 11.37 (which corresponds to the appearance of the 
sond chaotic attractor), numerical results show that $R_{1}$ is the only attractor. These rameter values imply transient instability. Also voltage collapse can take place.

From topological arguments it is clear that there must exist at least one unstable iit set on the boundary of each of the attractors $R_{i}, 1 \leqslant i \leqslant 3$. Denote the boundary $R_{i}$ by $\partial\left(R_{i}\right)$. We can test this by checking whether some component of this unstable inifold converges to $R_{i}$. We restrict ourselves to "some component" because an stable limit set could be on a boundary $\partial\left(R_{i}\right)$ which is "shared" by more than one ractor.

) For the example considered in $\S 3$, the following unstable limit sets, denoted , can be identified. $U_{1}$ is a type-1 unstable equilibrium point that persists till $Q_{\text {SNB }}$. tween $Q_{\mathrm{CFB}}$ and $Q_{\mathrm{UHB}}$, the unstable limit cycle emerging from the subcritical Hopf urcation at $Q_{U H B}$ is labelled as $U_{2}$. Since the two chaotic attractors are formed as a ult of two cascades of periodic doubling bifurcations, there is probably a countable $t$ infinite number of unstable limit cycles for $Q$ values belonging to two narrow nintersecting intervals which lie in between the values $Q_{\mathrm{PDB}_{1}}$ and $Q_{\mathrm{PDB}_{2}}$. Between нв and $Q_{\text {SHB }}, x_{s}$ turns into a type-2 unstable equilibrium point, which we label as . After $Q_{\mathrm{SHB}}$ only $U_{1}$ remains.

i) A component of the unstable manifold of $U_{1}$ converges to $R_{2}$ for $Q \leqslant 10.894$. e load demand $Q=10.894$ is where the chaotic attractor that occurs after PDB $_{1}$ dishes. At $Q=11.37$, another chaotic attractor makes its appearance. For $894 \leqslant Q \leqslant 11 \cdot 37$, the same component of the unstable manifold of $U_{1}$ converges $R_{1}$ (infinity). For $11 \cdot 37 \leqslant Q \leqslant 11 \cdot 38$, this component converges to $R_{3}$, which is now haotic attractor. It is very likely that, between these "switchings" of convergence various $R_{i}$, there are non-transversal scenarios such as trajectories connecting pairs unstable limit sets.

We consider such observations to be helpful in predicting the size of $A\left(x_{s}\right)$. For a-contracting diffeomorphisms, the unstable limit sets on the boundary are called essible saddles in Grebogi et al (1983). Accessible saddles could either be equilibrium $\mathrm{nts}$ or periodic orbits, on the boundary, that have stable and unstable directions. ien these accessible saddles themselves undergo bifurcation the size of $A\left(x_{s}\right)$ changes continuously. In Grebogi et al (1983) for example, the stability region of the Henon ractor experiences sudden changes in size at parameter values when homoclinic gencies, involving these accessible saddles, are shown to exist. The power system mple is not an area-contracting diffeomorphism; however the set of "accessible :dles" along with the parts of the singularity surface induced by algebraic constraints, y point out new ways to analyse $\partial A\left(x_{s}\right)$. Detailed calculations similar to those for simple swing equation considered in $\S 4$ should be carefully carried out for the ee-bus example studied in this paper.

is research was supported by the Electric Power Research Institute.

\section{ferences}

:d E H 1985 Singularly perturbed Hopf bifurcation. IEEE Trans. Circuits Syst. CAS-32: $1270-1280$

id E H, Alexander J C, Wang H, Hamdan A M A, Lee H C 1992 Dynamic bifurcations in a power system model exhibiting voltage collapse. University of Maryland, Technical Report no. TR 92-96 
Abed E H, Varaiya P P 1984 Nonlinear oscillations in power systems. Int.J. Electr. Power Energy Syst. 6: 37-43

Alexander JC 1986 Oscillatory solutions of a model system of nonlinear swing equations. Int. J. Electr. Power Energy Syst. 8: 130-136

Arapostathis A, Sastry S S, Varaiya P P i 982 Global analysis of swing dynamics. IEEE Trans. Circuits Syst. CAS-29: 673-679

Chen R L, Varaiya P P 1988 Degenerate Hopf bifurcation in power systems. IEEE Trans. Circuits Syst. C.AS-35: 818-824

Chiang H D 1989 Study of the existencc of energy functions for power systems with losses. IEEE Trans. Circuits Syst. CAS-36: 1423-1429

Chiang $\mathrm{H} \mathrm{D}$, Hirsch $\mathrm{M}, \mathrm{Wu} F \mathrm{~F} 1988$ Stability regions of nonlinear autonomous dynamical systems. IEEE Trans. Autom. Control 33: 16-27

Chiang H D, Liu C W, Varaiya P P, Wu F F, Lauby M G 1992 Cháos in a simple power system model. IEEE Winter Power Meeting Paper No. 92 WM 151-1 PWRS

DeMarco C L, Bergen A R 1987 A security measure for random load disturbances in nonlinear power system models. IEEE Trans. Circuits Syst. CAS-34: 1546-1557

Dobson I, Chiang H D 1989 Towards a theory of voltage collapse in electric power systems. Syst. Control Lett. 13: 253-262

Dobson I, Delchamps D F 1989 Basin boundaries in the pendulum with nonperiodic forcing. Proceedings of the 23rd Conference of Information Sciences and Systems, Baltimore

Fekih-Ahmed L, Chiang H D 1992 Analysis of voltage collapse in structure preserving models of the power system. Proceedings of the International Symposium on Circuits and Systems, San Diego, pp. 2525-2528

Grebogi C, Ott E, Yorke J A 1983 Crises, sudden changes in attractors, and transient chaos. Physica D7: 181-200

Guckenheimer J 1983 Persistent properties of bifurcations. Physica D7: 105-110

Guckenheimer J, Holmes P J 1986 Nonlinear oscillations, dynamical systems, and bifurcations of vector fields. Applied Mathematical Sciences, vol. 42 (Berlin: Springer-Verlag)

Kopell N, Washburn R B 1982 Chaotic motions in the two-degree-of-freedom swing equations. IEEE Trans. Circuits Syst. CAS-29: 738-746

Kwatny H G, Pasrija A K, Bahar L Y 1986 Static bifurcations in electric power systems: Loss of steady state and voltage collapse. IEEE Trans. Circuits Syst. CAS-33: 981-991

Milnor J 1963 Morse theory (Princeton, NJ: University Press)

Narasimhamurti N 1984 On the existence of energy functions for power systems with transmission losses. IEEE Trans. Circuits Syst. CAS-31: 199-203

Rajagopalan C, Sauer P W, Pai M A 1989 Analysis of voltage control systems exhibiting voltage collapse. Proceedings of the 28th Conference on Decision and Control (New York: IEEE Press) pp. 332-335

Salam F M A, Marsden J, Varaiya P P 1984 Arnold diffusion in swing equations of a power system. IEEE Trans. Circuits Syst. 30: 199-206

Sastry S S, Desoer C A 1981 Jump behaviour of circuits and systems. IEEE Trans. Circuits Syst. 28: $1109-1124$

Takens F 1976 Constrained equations: a study of implicit differential equations and their discontinuous solutions. Lecture Notes in Mathematics, 525 (Berlin: Springer-Verlag)

Thom R 1975 Stability and morphogenesis (Transl. by D H Fowler) (Paris: Addison-Wesley)

Tsolas N A, Arapostathis A, Varaiya P P 1985 A structure preserving energy function for power system transient stability anlaysis. IEEE Trans. Circuit Syst. CAS-32: 1041 1049

Varaiya P P, Wu F F, Chiang H D 1990 Bifurcation and chaos in power systems: A survey. ERL/UCB Memo no. M90/98, University of California, Berkeley

Varghese M, Chiang H D, Thorp J S 1993 Computer algorithms in power systems: From constructive methods to truncated fractals. IEEE Trans. Educ. 36: 36-41

Venkatasubramanian V, Schattler H, Zaborsky J 1992 A stability theory for differential algebraic systems such as the power system. Proceedings of the International Symposium of Circuits and Systems (New York: IEEE Press) pp. 2517-2520

Wang H, Abed E H 1992 Bifurcation control of chaotic dynamical systems. Proceedings of the Nonlinear Control Systems Design Symposium (Paris: IFAC Press) pp. 57-62

Zaborsky J, Huang G, Zheng B, Leung T C 1988 On the phase portraits of a class of large nonlinear dynamical systems such as the power system. IEEE Trans. Autom. Control 33: 4-15 